DOI 10.4171/JEMS/293

Philippe Eyssidieux $\cdot$ Carlos Simpson

\title{
Variations of mixed Hodge structure attached to the deformation theory of a complex variation of Hodge structures
}

Received April 2, 2009 and in revised form November 26, 2009

\begin{abstract}
Let $X$ be a compact Kähler manifold, $x \in X$ be a base point and $\rho: \pi_{1}(X, x) \rightarrow$ $G L_{N}(\mathbb{C})$ be the monodromy representation of a $\mathbb{C}$-VHS. Building on Goldman-Millson's classical work, we construct a mixed Hodge structure on the complete local ring of the representation variety at $\rho$ and a variation of mixed Hodge structures whose monodromy is the universal deformation of $\rho$.
\end{abstract}

Let $X$ be a compact connected Kähler manifold, $x \in X$ and $\Gamma=\pi_{1}(X, x)$. Let $\rho: \Gamma \rightarrow G L_{N}(\mathbb{C})$ be a finite-dimensional semisimple representation. We assume $\rho$ to be the monodromy of a given polarized $\mathbb{C}$-VHS $\left(\mathbb{V}_{\rho}, \mathcal{F}^{\bullet}, \overline{\mathcal{G}}^{\bullet}, S\right)$. Without loss of generality, we assume its weight is 0 . If $\rho$ is not irreducible then several distinct polarizations could be chosen; we fix one once and for all. In the introduction, we fix an isomorphism $\mathbb{V}_{\rho, x} \cong \mathbb{C}^{N}$.

The variety $R\left(\Gamma, G L_{N}\right)$ of representations of $\Gamma$ in $G L_{N}$ [LuMa85] may be viewed as an affine scheme over $\mathbb{Z}$ but we will only consider it as an affine scheme over $\mathbb{C}$. The group $G L_{N}$ acts algebraically on $R\left(\Gamma, G L_{N}\right)$ by conjugation. The conjugation orbit $\Omega_{\rho}$ of $\rho$ is a closed smooth algebraic subvariety of $R\left(\Gamma, G L_{N}\right)$, considered as a subscheme endowed with the induced reduced structure.

Denote by $R\left(\Gamma, G L_{N}\right)_{\rho}$ the formal local scheme which is the germ at $[\rho]$ of $R\left(\Gamma, G L_{N}\right)$. Similarly, denote by $\hat{\Omega}_{\rho}$ the germ of $\Omega_{\rho}$ at $[\rho]$; it is a closed formal subscheme of $R\left(\Gamma, G L_{N}\right)_{\rho}$. Let $\left(\hat{\mathcal{O}}_{\rho}, \mathfrak{m}\right)$ be the complete local ring of $[\rho] \in R\left(\Gamma, G L_{N}\right)(\mathbb{C})$ so that

$$
R\left(\Gamma, G L_{N}\right)_{\rho}=\operatorname{Spf}\left(\hat{\mathcal{O}}_{\rho}\right) .
$$

We construct on $\hat{\mathcal{O}}_{\rho}$ a natural mixed Hodge structure whose weight filtration is given by powers of the ideal of $\hat{\Omega}_{\rho}$. It is infinite-dimensional, so it is easier to work with the natural quotients by powers of the maximal ideal. Let $n$ be a strictly positive integer and denote by $\mathcal{O}_{\rho \mid n}:=\mathcal{\mathcal { O }}_{\rho} / \mathfrak{m}^{n}$ the Artin algebra of the $(n-1)$-th infinitesimal neighborhood of $[\rho]$.

P. Eyssidieux: Institut Fourier, UMR 5582 CNRS, Université Joseph Fourier, Université de Grenoble I, 100 rue des Maths, BP 74, 38402 Saint Martin d'Hères Cedex, France; e-mail: philippe.eyssidieux@ujf-grenoble.fr, URL: http://www-fourier.ujf-grenoble.fr/ eyssi/

C. Simpson: CNRS, Laboratoire J. A. Dieudonné, UMR 6621, Université de Nice-Sophia Antipolis, 06108 Nice, Cedex 2, France; e-mail: carlos@ unice.fr, URL: http://math.unice.fr/ carlos/ 
A direct consequence of the formality result of [GoMi88] is the construction of a split $\mathbb{R}-$ MHS on $\mathcal{O}_{\rho \mid n}$. This construction is essentially included in [Pri04, p. 5] (see also [Pri06]). The choices made in the construction, the fact that the MHS is split, and the fact that the outcome of the construction does not depend on $x \in X$, make it clear that this split MHS is only the approximation $G r_{W}$ of the true object. Something more subtle must be done to confirm the conjecture in [Sim97] that there should be a (not necessarily split) MHS on $\mathcal{O}_{\rho \mid n}$ depending explicitly on $x$. This is done in Section 3, where we show in Theorem 3.24 that the finite-dimensional vector spaces $\mathcal{O}_{\rho \mid n}$ carry natural mixed Hodge structures. They are compatible with the inverse system of surjections $\mathcal{O}_{\rho \mid n+1} \rightarrow \mathcal{O}_{\rho \mid n}$ giving a pro-MHS on $\hat{\mathcal{O}}_{\rho}$ whose $G r_{W}$ is the split MHS referred to above.

Unfortunately, in general the powers of the maximal ideal $\mathfrak{m}^{n}$ will not constitute the weight filtration, and indeed the MHS on $\hat{\mathcal{O}}_{\rho}$ obtained by passing to the limit is rather unpleasant since it has infinite-dimensional weight subquotients. This distinguishes the present situation from the special case when $\rho$ is the identity representation. In general, instead of considering the maximal ideal defining $[\rho]$ itself, we should consider the ideal of the orbit. Let $\mathrm{j} \subset \hat{\mathcal{O}}_{\rho}$ be the prime ideal defining $\hat{\Omega}_{\rho}$. For $n \geq k \geq 0$, let $W_{-k} \mathcal{O}_{\rho \mid n} \simeq$ $\mathfrak{j}^{k} / \mathfrak{j}^{k} \cap \mathfrak{m}^{n}$ be the image of $\mathfrak{j}^{k}$ in $\mathcal{O}_{\rho \mid n}$. These subspaces will form the weight filtration on $\mathcal{O}_{\rho \mid n}$.

By looking at a natural slice, we can get back to the situation where the weight filtration coincides with the filtration by powers of the maximal ideal. Let $T$ be the formal germ at the origin of the homogeneous quadratic cone defined by the zeroes of the obstruction map

$$
\operatorname{obs}_{2}: S^{2} H^{1}\left(X, \operatorname{End}\left(\mathbb{V}_{\rho}\right)\right) \rightarrow H^{2}\left(X, \operatorname{End}\left(\mathbb{V}_{\rho}\right)\right)
$$

and let $\hat{\mathcal{O}}_{T}$ be its complete local coordinate ring so $T=\operatorname{Spf}\left(\hat{\mathcal{O}}_{T}\right)$. Note that $\hat{\mathcal{O}}_{T}$ is just the quotient of a power series ring by the ideal generated by the image of $\mathrm{obs}_{2}{ }^{t}$. By formality [GoMi88], the Kuranishi obstruction map is equivalent to its quadratic part and $T$ is isomorphic to the Kuranishi deformation space of $\rho$.

One can slightly revise the statements of [GoMi88] and give the construction of an isomorphism of formal germs:

$$
G M^{c}: R\left(\Gamma, G L_{N}\right)_{\rho} \rightarrow \hat{\Omega}_{\rho} \times T
$$

which we call the preferred Goldman-Millson isomorphism (Definition 2.11). The main theorem of [GoMi88] asserts that an abstract isomorphism between these two formal germs exists.

The original construction is not quite canonical, and we have tried to make the choices made there as explicit as possible. This is the rationale for the length of Section 2. It turns out that there is no good reason to give any kind of privilege to this preferred isomorphism. Two other very nice Goldman-Millson isomorphisms $G M^{\prime}$ and $G M^{\prime \prime}$ can be constructed and the rest of the article can in retrospect be understood as the study of their interplaymore concretely than in [Pri09].

The isomorphism $G M^{c}$ provides $T$ with an inclusion in $R\left(\Gamma, G L_{N}\right)_{\rho}$ making it a slice transverse to the orbit $\hat{\Omega}_{\rho}$. The complete local ring $\hat{\mathcal{O}}_{T}$ is somewhat better behaved than $\hat{\mathcal{O}}_{\rho}$ in certain respects. In particular, the graded Artin local ring $\left(\hat{\mathcal{O}}_{T} / \mathfrak{m}^{n}\right)$ has a 
canonical split MHS with finite-dimensional weight subquotients and weight filtrations given by the powers of the maximal ideal. On the other hand, beyond the simple split structure, its mixed Hodge theory is a bit more messy than that of $\hat{\mathcal{O}}_{\rho}$ because of the choices involved in the isomorphisms $G M$.

The restriction to $T \subset \operatorname{Spf}\left(\hat{\mathcal{O}}_{\rho}\right)$ of the tautological representation $\Gamma \rightarrow G L_{N}\left(\hat{\mathcal{O}}_{\rho}\right)$ is a natural local system of $\hat{\mathcal{O}}_{T}$-modules over $X$. Restricting to a finite infinitesimal neighborhood, we get a representation denoted $\rho_{T, n}: \Gamma \rightarrow G L_{N}\left(\hat{\mathcal{O}}_{T} / \mathfrak{m}^{n}\right)$ or equivalently a locally free rank $N\left(\hat{\mathcal{O}}_{T} / \mathfrak{m}^{n}\right)$-local system $\mathbb{V}_{n}$ on $X$. Since $\rho_{T, n}(\Gamma)$ preserves the decreasing filtration defined at level $k$ by $\mathfrak{m}^{k}\left(\hat{\mathcal{O}}_{T} / \mathfrak{m}^{n}\right)^{N} \subset\left(\hat{\mathcal{O}}_{T} / \mathfrak{m}^{n}\right)^{N}$, this filtration induces on $\mathbb{V}_{n}$ an increasing filtration indexed by the nonpositive integers $W_{-n} \subset \cdots \subset W_{-k} \subset W_{-k+1} \subset$ $\cdots \subset W_{0}=\mathbb{V}_{n}$. That is to say, this weight filtration is defined by powers of the maximal ideal of $\left(\hat{\mathcal{O}}_{T} / \mathfrak{m}^{n}\right)$.

Our original motivation was to give this local system a structure of variation of mixed Hodge structure over $X$. Such a VMHS helps in the proof of the linear Shafarevich conjecture [E-R09]. The main result is the following basic fact:

Theorem 1. The filtration $W_{\bullet}$ is the weight filtration of a $\mathbb{C}$-variation of mixed Hodge structures on $X$ whose monodromy representation is $\rho_{T, n}: \Gamma \rightarrow G L_{N}\left(\hat{\mathcal{O}}_{T} / \mathfrak{m}^{n}\right)$.

The proof is given in the first part of Section 3. In the second part of that section, after Theorem 3.24 concerning the mixed Hodge structure on $\mathcal{O}_{\rho \mid n}$, we give in Theorem 3.26 the analogue of Theorem 1 saying that the full tautological representation $\Gamma \rightarrow G L_{N}\left(\mathcal{O}_{\rho \mid n}\right)$ underlies a $\mathbb{C}$-VMHS compatible with the action of the MHS $\mathcal{O}_{\rho \mid n}$.

The VMHS of Theorems 1 and 3.26 are closely related to-but somewhat different from-those constructed in [Ha98, Theorem 13.10 p. 82]. The explicit nature of the construction in Theorem 1 makes these VMHS easier to use in applications such as [E-R09]. Understanding the relationship with [Ha98] is left as an interesting problem for the future. A similar and related problem will be to investigate the behavior of the MHS on $\mathcal{O}_{\rho \mid n}$ and the VMHS of Theorem 1 as the basepoint $x$ moves around in $X$.

The twistor picture of [Sim97] should give a way of characterizing geometrically the MHS on $\mathcal{O}_{\rho \mid n}$ and the tautological VMHS of Theorem 3.26. Very briefly, the MHS on $\mathcal{O}_{\rho \mid n}$ leads to a family of complete local rings indexed by $\mathbb{P}^{1}$ via the Rees constructions at 0 and $\infty$ using the Hodge and anti-Hodge filtrations respectively. The resulting formal scheme, supported on and mapping to $\mathbb{P}^{1}$, should be naturally isomorphic to the formal completion of the Hitchin twistor space of representations [Fuj91], along the preferred section corresponding to the VHS $\rho$. We do not verify this compatibility here as that would go beyond our present scope.

In contrast, the slice $T$ not being quite canonical inside $R\left(\Gamma, G L_{N}\right)_{\rho}$, the possibility of creating a VMHS as is done in Theorem 1 on the tautological local system restricted over $T$ is new and unexpected. We do not at present have any geometric interpretation or characterization in terms of twistor geometry.

In Section 4 we generalize these results to the case of representations in a complex reductive algebraic group $G$, and take the occasion to give more precise information on some aspects. Everything is summed up in Theorem 4.1 which we now describe informally. Suppose $\sigma: \Gamma \rightarrow G(\mathbb{C})$ is a semisimple representation whose associated Higgs 
bundle is a fixed point of the $\mathbb{C}^{*}$-action on $M_{D o l}(X, G)$ [Sim94], in other words $\sigma$ comes from a principal variation of Hodge structure with structure group $G$. Let $R(\Gamma, G) / \mathbb{C}$ be the affine scheme parametrizing the representations of $\Gamma$ with values in $G$ endowed with the action of $G$ by conjugation; and denote by $\hat{\Omega}_{\sigma}$ the formal germ at $[\sigma]$ of the orbit of $\sigma$. Let $\hat{\mathcal{O}}_{\sigma}$ denote the formal completion of the local coordinate ring of $R(\Gamma, G)$ at $\sigma$, so the formal infinitesimal neighborhood of $\sigma$ is $\operatorname{Spf}\left(\hat{\mathcal{O}}_{\sigma}\right)$.

As before, let $T=\operatorname{Spf}\left(\hat{\mathcal{O}}_{T}\right)$ be the formal germ at the origin of the homogeneous quadratic cone attached to the obstruction map

$$
S^{2} H^{1}\left(X, \operatorname{ad}_{\sigma}\right) \rightarrow H^{2}\left(X, \operatorname{ad}_{\sigma}\right) .
$$

We will show in Theorem 4.1 that for every $n$, the truncated coordinate ring $\hat{\mathcal{O}}_{\sigma} / \mathfrak{m}^{n}$ carries a canonical functorial $\mathbb{C}$-MHS. Along the way, we again construct a preferred Goldman-Millson isomorphism $G M^{c}: \operatorname{Spf}\left(\hat{\mathcal{O}}_{\sigma}\right) \rightarrow \hat{\Omega}_{\sigma} \times T$.

To generalize Theorem 1 in this case, choose a rational representation $\alpha: G \rightarrow G L_{N}$ and let $\sigma_{n}: \Gamma \rightarrow G\left(\hat{\mathcal{O}}_{T} / \mathfrak{m}^{n}\right)$ be the tautological representation. Denote by $\mathbb{V}_{\alpha, \sigma}$ the local system of $\left(\hat{\mathcal{O}}_{T} / \mathfrak{m}^{n}\right)$ free modules on $X$ attached to the representation $\alpha \circ \sigma: \Gamma \rightarrow$ $G L_{N}\left(\hat{\mathcal{O}}_{T} / \mathfrak{m}^{n}\right)$.

The main statement of Theorem 4.1 says that the $\mathbb{C}$-local system underlying $\mathbb{V}_{\alpha, \sigma}$ is the holonomy of a graded polarizable VMHS whose weight filtration is given by

$$
W_{-k} \mathbb{V}_{\alpha, \sigma}=\mathfrak{m}^{k} \cdot \mathbb{V}_{\alpha, \sigma}, \quad k=0, \ldots, n .
$$

\section{Basic definitions in mixed Hodge theory}

The following definitions are well-known to experts. We nevertheless recall them for the reader's convenience.

\section{1. $\mathbb{C}-M H S$}

Definition 1.1. Let $w \in \mathbb{Z}$ be an integer. A complex (finite-dimensional) Hodge structure (for short, HS) of weight $w$ is a triple $\left(V, F^{\bullet}, \bar{G}^{\bullet}\right)$ where $F^{\bullet}, \bar{G}^{\bullet}$ are $w$-opposed decreasing biregular filtrations on the (finite-dimensional) $\mathbb{C}$-vector space $V$, that is, $G r_{F}^{p} G r_{\bar{G}}^{q}(V)$ $=0$ if $p+q \neq w$

If $V$ is finite-dimensional, a hermitian form $S$ on $V$ polarizes the Hodge structure if, using the usual definition $H^{p, q}=F^{p} \cap \bar{G}^{q}$ for $p+q=w$, the decomposition $V=\bigoplus_{p+q=w} H^{p, q}$ is $S$-orthonormal and $\left.(-1)^{p+w} S\right|_{H, q}>0$.

A complex (finite-dimensional) mixed Hodge structure (for short, $\mathbb{C}-\mathrm{MHS}$ ) is a quadruple $\left(V, F^{\bullet}, \bar{G}^{\bullet}, W_{\bullet}\right)$ where $F^{\bullet}, \bar{G}^{\bullet}$ decreasing biregular filtrations and $W_{\bullet}$ is an increasing filtration on the (finite-dimensional) $\mathbb{C}$-vector space $V$ such that the filtrations induced by $F^{\bullet}, \bar{G}^{\bullet}$ on $G r_{k}^{W}(V)$ give rise to a weight $k$ complex HS.

A complex (finite-dimensional) $H S$ defined over the reals is an $\mathbb{R}$-vector space $V_{\mathbb{R}}$ with a $\mathbb{C}$-Hodge structure $\left(V_{\mathbb{C}}, F^{\bullet}, \bar{F}^{\bullet}\right)$ on its complexification, such that $\bar{F}^{\bullet}$ is the complex conjugate of $F^{\bullet}$. A real polarization is a real symmetric or antisymmetric bilinear form whose associated hermitian form is polarizing in the above sense. 
A complex (finite-dimensional) $M H S$ defined over the reals is a $\mathbb{R}$-vector space $V_{\mathbb{R}}$ with a $\mathbb{C}$-mixed Hodge structure $\left(V_{\mathbb{C}}, F^{\bullet}, \bar{F}^{\bullet}, W_{\bullet}\right)$ on its complexification, such that $W_{\bullet}$ is the complexification of an increasing filtration of $V_{\mathbb{R}}$ and $\bar{F}^{\bullet}$ is the complex conjugate of $F^{\bullet}$.

Proposition 1.2 ([Del71]). The category of complex mixed Hodge structures is abelian. For the usual tensor product and duality functor the category of complex mixed Hodge structures is tannakian. The $\mathbb{C}$-Hodge structures form a full abelian and tannakian subcategory.

The following definition is thus natural:

Definition 1.3. A $\mathbb{C}$-Hodge (positively) graded Lie algebra is a complex graded vector space $\bigoplus_{k \geq 0} L^{k}$, each $L^{k}$ being endowed with a $\mathbb{C}$-Hodge structure of weight $k$, with a graded Lie algebra bracket respecting the Hodge structure.

A Hodge Lie algebra is a real finite-dimensional Lie algebra $\mathfrak{g}$ such that $\mathfrak{g}_{\mathbb{C}}$ carries a $\mathbb{C}$-Hodge structure of weight zero defined over $\mathbb{R}$ respected by the Lie bracket.

Example 1.4. The Lie algebra of a group of Hodge type [Sim92] is a Hodge Lie algebra.

Lemma 1.5. Let $\mathbb{M}=\left(V, F^{\bullet}, \bar{G}^{\bullet}, W_{\bullet}\right)$ be a mixed Hodge structure. Let $u \in G L(V)$ such that $u-\operatorname{Id}_{V} \in W^{-1} \operatorname{End}(V)$. Then $\mathbb{M}_{u}=\left(V, F^{\bullet}, u\left(\bar{G}^{\bullet}\right), W_{\bullet}\right)$ is a MHS such that $G r_{\bullet}^{W}\left(\mathbb{M}_{u}\right)=G r_{\bullet}^{W}(\mathbb{M})$ as split mixed Hodge structures.

Proof. This is immediate since the filtration induced by $\bar{G}^{\bullet}$ and $u\left(\bar{G}^{\bullet}\right)$ on $G r_{\bullet}^{W}(V)$ coincide. Indeed, $u$ preserves $W^{\bullet}$ and induces the identity on $G r_{\bullet}^{W}(V)$.

Lemma 1.6. Let $\left(V, F^{\bullet}, \bar{G}^{\bullet}, W_{\bullet}\right)$ be a finite-dimensional complex vector space with three biregular filtrations having a fourth biregular filtration $U^{\bullet}$ such that the filtrations induced by $F^{\bullet}, \bar{G}^{\bullet}, W_{\bullet}$ on every $G_{U}^{r}(V)$ give rise to a mixed Hodge structure. Then $\left(V, F^{\bullet}, \bar{G}^{\bullet}, W_{\bullet}\right)$ is itself a mixed Hodge structure.

Proof. There should be a reference for this claim in the MHS literature, but here is a short proof. It suffices by induction to prove this for a 2-step filtration, which comes down to the following statement: if $U$ is a vector space with three filtrations $W, F, G$, and $U^{\prime} \subset U$ is a subspace with quotient denoted $U^{\prime \prime}:=U / U^{\prime}$, and if we suppose that the induced filtrations on $U^{\prime}$ and $U^{\prime \prime}$ are MHS, then the filtrations on $U$ form a MHS.

In turn, this can be seen by Penacchio's interpretation [Pen02]: given three filtrations we get a bundle on $\mathbb{P}^{2}$, and they form a MHS if and only if the bundle is $\mu$-semistable of slope 0 . The subspace $U^{\prime}$ with its filtrations corresponds to a locally free subsheaf, and $U^{\prime \prime}$ is the reflexive sheaf associated to the quotient sheaf. We get a short exact sequence of sheaves outside of codimension 2, and in this case if the kernel and cokernel are $\mu$ semistable of slope 0 then so is the middle bundle.

Suppose $A$ is an artinian local ring, with increasing filtration $W$ and decreasing filtrations $F$ and $G$, all compatible with the algebra structure. Let $\mathbf{m}$ denote the maximal ideal and let $M^{k}:=\mathbf{m}^{k}$ be the decreasing filtration of $A$ by powers of $n$. Let $n$ be the smallest integer with $M^{n}=0$. 
Set $V:=M^{1} / M^{2}=G r_{M}^{1}(A)$, which is the dual of the Zariski tangent space of $A$. Note that the filtrations $W, F$ and $G$ induce filtrations given by the same letter on the associated graded pieces $G r_{M}^{k}(A)$, in particular on $V$. The associated-graded algebra $G r_{M}^{\bullet}(A)=\bigoplus_{k=0}^{n-1} G r_{M}^{k}(A)$ is generated by the piece in degree 1 which is $V$. This means that we have surjections

$$
\operatorname{Sym}^{k}(V) \stackrel{\mu^{k}}{\rightarrow} G r_{M}^{k}(A) \rightarrow 0 .
$$

The source $\operatorname{Sym}^{k}(V)$ has three filtrations obtained from the symmetric product operation applied to the filtrations of $V$, whereas the target $G r_{M}^{k}(A)$ has induced filtrations as stated above. The map $\mu^{k}$ comes from the algebra structure so it preserves the three filtrations.

Proposition 1.7. Suppose that the above data satisfy the following hypotheses:

(1) $\operatorname{Spec}(A)$ is the $n$-th neighborhood of the origin in a quadratic cone,

(2) the filtrations $W, F, G$ induce a complex MHS on $V$, which we use also to give a $\mathbb{C}-M H S$ on $\operatorname{Sym}^{k}(V)$,

(3) the kernel $K$ of the map $\mu^{2}: \operatorname{Sym}^{2}(V) \rightarrow G r_{M}^{2}(A)$ is a sub-C-MHS of $\operatorname{Sym}^{2}(V)$,

(4) for each $k$, the filtrations induced by $W, F$ and $G$ on $G_{M}^{k}(A)$ are the same as the quotient filtrations induced by the map $\mu^{k}$ from the filtrations on $\operatorname{Sym}^{k}(V)$, in other words $\mu^{k}$ strictly preserves the filtrations.

Then $W, F, G$ induce a $\mathbb{C}$-MHS on $A$ and $M^{\bullet}$ is a filtration by sub-C-MHS's.

Proof. Using condition (1) we get exact sequences

$$
K \otimes \operatorname{Sym}^{k-2}(V) \rightarrow \operatorname{Sym}^{k}(V) \rightarrow G r_{M}^{k}(A) \rightarrow 0 .
$$

By condition (3), $K$ is a sub-C-MHS of $\operatorname{Sym}^{2}(V)$, so the map $K \otimes \operatorname{Sym}^{k-2}(V) \rightarrow$ $\operatorname{Sym}^{k}(V)$ is a morphism of $\mathbb{C}$-MHS. Hence, its cokernel is a $\mathbb{C}$-MHS; then condition (4) says that $G r_{M}^{k}(A)$ with its triple of filtrations is equal to this cokernel, so $G r_{M}^{k}(A)$ is a $\mathbb{C}$-MHS. Now, apply Lemma 1.6.

\section{2. $\mathbb{C}-V M H S$}

Definition 1.8. A $\mathbb{C}-V H S$ (polarized complex variation of Hodge structures) on $X$ of weight $w \in \mathbb{Z}$ is a 5-tuple $\left(X, \mathbb{V}, \mathcal{F}^{\bullet}, \overline{\mathcal{G}}^{\bullet}, S\right)$ where:

(1) $\mathbb{V}$ is a local system of finite-dimensional $\mathbb{C}$-vector spaces,

(2) $S$ a nondegenerate flat sesquilinear pairing on $\mathbb{V}$,

(3) $\mathcal{F}^{\bullet}=\left(\mathcal{F}^{p}\right)_{p \in \mathbb{Z}}$ is a biregular decreasing filtration of $\mathbb{V} \otimes_{\mathbb{C}} \mathcal{O}_{X}$ by locally free coherent analytic sheaves such that $d^{\prime} \mathcal{F}^{p} \subset \mathcal{F}^{p-1} \otimes \Omega_{X}^{1}$,

(4) $\overline{\mathcal{G}}^{\bullet}=\left(\overline{\mathcal{G}}^{q}\right)_{q \in \mathbb{Z}}$ is a biregular decreasing filtration of $\mathbb{V} \otimes_{\mathbb{C}} \mathcal{O}_{\bar{X}}$ by locally free coherent antianalytic sheaves such that $d^{\prime \prime} \overline{\mathcal{G}}^{p} \subset \overline{\mathcal{G}}^{p-1} \otimes \Omega_{\bar{X}}^{1}$,

(5) for every point $x \in X$ the fiber at $x,\left(\mathbb{V}_{x}, \mathcal{F}_{x}^{\bullet}, \overline{\mathcal{G}}_{x}^{\bullet}\right)$, is a $\mathbb{C}$-HS polarized by $S_{x}$.

This definition is easily seen to be equivalent to that given by [Sim88]. 
Example 1.9. Let $\rho: \pi_{1}(X, x) \rightarrow G L\left(\mathbb{V}_{\rho, x}\right)$ be the monodromy representation underlying a $\mathbb{C}$-VHS and $\mathbb{V}_{\rho}$ be the corresponding local system. Then $\operatorname{ad}_{\rho}=\operatorname{End}\left(\mathbb{V}_{\rho}\right)$ is a local system of Lie algebras that underlies a $\mathbb{C}$-VHS.

Then $H^{\bullet}\left(X\right.$, End $\left.\left(\mathbb{V}_{\rho}\right)\right)$ is a $\mathbb{C}$-Hodge graded Lie algebra for the bracket obtained by composing the usual cup product

$$
H^{\bullet}\left(X, \operatorname{End}\left(\mathbb{V}_{\rho}\right)\right) \otimes H^{\bullet}\left(X, \operatorname{End}\left(\mathbb{V}_{\rho}\right)\right) \rightarrow H^{\bullet}\left(X, \operatorname{End}\left(\mathbb{V}_{\rho}\right) \otimes \operatorname{End}\left(\mathbb{V}_{\rho}\right)\right)
$$

with the cohomology operation

$$
H^{\bullet}\left(X, \operatorname{End}\left(\mathbb{V}_{\rho}\right) \otimes \operatorname{End}\left(\mathbb{V}_{\rho}\right)\right) \rightarrow H^{\bullet}\left(X, \operatorname{End}\left(\mathbb{V}_{\rho}\right)\right)
$$

induced by the Lie bracket $\operatorname{End}\left(\mathbb{V}_{\rho}\right) \otimes \operatorname{End}\left(\mathbb{V}_{\rho}\right) \rightarrow \operatorname{End}\left(\mathbb{V}_{\rho}\right)$.

The $\mathbb{C}$-Hodge structure on the cohomology of a $\mathbb{C}$-VHS can be constructed by a straightforward adaptation of the Deligne-Zucker argument [Zu79], which is written for the real case.

The following definition is a slight generalization of the definition in [Usu83].

Definition 1.10. A $\mathbb{C}$-VMHS on $X$ is a 6-tuple $\left(X, \mathbb{V}, \mathbb{W} \bullet, \mathcal{F}^{\bullet}, \overline{\mathcal{G}}^{\bullet},\left(S_{k}\right)_{k \in \mathbb{Z}}\right)$ where:

(1) $\mathbb{V}$ is a local system of finite-dimensional $\mathbb{C}$-vector spaces,

(2) $\mathbb{W}_{\bullet}=\left(\mathbb{W}_{k}\right)_{k \in \mathbb{Z}}$ is a decreasing filtration of $\mathbb{V}$ by local subsystems,

(3) $\mathcal{F}^{\bullet}=\left(\mathcal{F}^{p}\right)_{p \in \mathbb{Z}}$ is a biregular decreasing filtration of $\mathbb{V} \otimes_{\mathbb{C}} \mathcal{O}_{X}$ by locally free coherent analytic sheaves such that $d^{\prime} \mathcal{F}^{p} \subset \mathcal{F}^{p-1} \otimes \Omega_{X}^{1}$,

(4) $\overline{\mathcal{G}}^{\bullet}=\left(\overline{\mathcal{G}}^{q}\right)_{q \in \mathbb{Z}}$ is a biregular decreasing filtration of $\mathbb{V} \otimes_{\mathbb{C}} \mathcal{O}_{\bar{X}}$ by locally free coherent antianalytic sheaves such that $d^{\prime \prime} \overline{\mathcal{G}}^{p} \subset \overline{\mathcal{G}}^{p-1} \otimes \Omega_{\bar{X}}^{1}$,

(5) for all $x \in X$ the stalk $\left(\mathbb{V}_{x}, \mathbb{W}_{\bullet, x}, \mathcal{F}_{x}^{\bullet}, \overline{\mathcal{G}}_{x}^{\bullet}\right)$ is a $\mathbb{C}$-MHS,

(6) $S_{k}$ is a flat sesquilinear nondegenerate pairing on $G r_{k}^{\mathbb{W}}(\mathbb{V})$,

(7) $\left(X, G r_{k}^{\mathbb{W}}(\mathbb{V}), \mathcal{F}^{\bullet} G r_{k}^{\mathbb{W}}(\mathbb{V}) \otimes_{\mathbb{C}} O_{X}, \overline{\mathcal{G}}^{\bullet} G r_{k}^{\mathbb{W}}(\mathbb{V}) \otimes_{\mathbb{C}} O_{\bar{X}}, S_{k}\right)$ is a $\mathbb{C}$-VHS of weight $k$.

A $\mathbb{C}$-VMHS is uniquely determined by its monodromy (as a $\mathbb{W}$-filtered representation of $\pi_{1}(X, x)$ ) and the MHS $\left(\mathbb{V}_{x}, \mathbb{W}_{x}^{\bullet}, \mathcal{F}_{x}^{\bullet}, \overline{\mathcal{G}}_{x}^{\bullet}\right.$ ) (rigidity theorem, cf. [HaZu87, p. 85, (1.7)c]) and the references therein).

To be consistent with earlier terminology, we could also have called $\mathbb{C}$-VHS polarized $\mathbb{C}$-VHS (resp. $\mathbb{C}$-VMHS graded polarized $\mathbb{C}$-VMHS).

Lemma 1.11. Let $E^{\bullet}=E^{\bullet}\left(X\right.$, End $\left.\left(\mathbb{V}_{\rho}\right)\right)$ be the $C^{\infty}$-de Rham complex of the $\mathbb{C}$-VHS attached to $\operatorname{ad}_{\rho}=\operatorname{End}\left(\mathbb{V}_{\rho}\right)$. It is endowed with the usual Hodge filtrations, and the usual Lie bracket preserves the Hodge filtrations. Endow $E^{\bullet}$ with the shifted (décalée) weight filtration defined by $W_{i+1} E^{i}=E^{i}, W_{i} E^{i}=\operatorname{ker}(d), W_{i-1} E^{i}=0$. We have the familiar conditions from [Del75]:

- The differential of $E^{\bullet}$ is strictly compatible with the two filtrations induced by $F^{\bullet}$ and $\bar{G}^{\bullet}$.

- The induced filtrations on $H^{k}\left(X, \operatorname{End}\left(\mathbb{V}_{\rho}\right)\right)$ give a $\mathbb{C}$-Hodge structure of weight $k$.

The Lie bracket induces on the cohomology $H^{\bullet}\left(X, \operatorname{End}\left(\mathbb{V}_{\rho}\right)\right)=H^{\bullet}\left(E^{\bullet}\right)$ the structure of a Hodge graded Lie algebra described in Example 1.9. 


\section{Goldman-Millson theory}

In what follows, we fix a $\mathbb{C}$-VHS $\left(\mathbb{V}_{\rho}, \mathcal{F}^{\bullet}, \overline{\mathcal{G}}^{\bullet}, S\right)$ on $X$ and denote by $\rho: \pi_{1}(X, x) \rightarrow$ $G L\left(\mathbb{V}_{\rho, x}\right)$ its holonomy.

In [GoMi88] the complete local ring $\hat{\mathcal{O}}_{\rho}$ is described rather precisely. We shall review this theory, pointing out some additional facts easily deduced from this classical reference. We shall also review some additions made by these authors in [GoMi90].

\subsection{General representability criteria in Deligne-Goldman-Millson theory}

2.1.1. The Deligne-Goldman-Millson groupoid attached to a dgla. Given a small groupoid $\mathcal{G}$, we will denote by Iso $\mathcal{G}$ the set of its isomorphism classes, i.e. the quotient of the set of objects by the equivalence relation induced by the arrows. ${ }^{1}$ Iso is a covariant functor from the category of small groupoids to the category of sets.

Let $L^{\bullet}$ be a dgla, graded by the nonnegative integers and defined over $\mathbb{C}$. We will assume finite-dimensionality of its cohomology objects. Let $(A, \mathfrak{m})$ be an Artin local $\mathbb{C}$ algebra (we assume $A / \mathfrak{m}=\mathbb{C}$ ). Then one defines the small groupoid $\operatorname{DGM}\left(L^{\bullet}, A\right)$ by:

$$
\begin{aligned}
\operatorname{Obj} D G M\left(L^{\bullet}, A\right) & =\left\{\alpha \in L^{1} \otimes \mathfrak{m} \mid d \alpha+\frac{1}{2}[\alpha, \alpha]=0\right\}, \\
\operatorname{Hom}_{D G M\left(L^{\bullet}, A\right)}(\alpha, \beta) & =\left\{\lambda \in L^{0} \otimes \mathfrak{m} \mid \exp (\lambda) \alpha=\beta\right\},
\end{aligned}
$$

where $\exp (\lambda) \alpha$ denotes the gauge transformation $e^{\lambda} \circ \alpha \circ e^{-\lambda}-d\left(e^{\lambda}\right) \circ e^{-\lambda}$.

This gives a covariant functor $\operatorname{DGM}\left(L^{\bullet},-\right)$ on the category Art of $\mathbb{C}$-Artin local rings with values in the category of small groupoids. Let us describe this functor. In what follows, the generic object of Art will be denoted by $A$, and $\mathfrak{m}$ will stand for the maximal ideal of $A$. The groupoid $\operatorname{DGM}\left(L^{\bullet}, A\right)$ is the transformation groupoid ${ }^{2}$ associated to the set-theoretic action of the simply connected nilpotent (infinite-dimensional) Lie group $\exp \left(L^{0} \otimes \mathfrak{m}\right)$ on $\operatorname{Obj} D G M\left(L^{\bullet}, A\right) .^{3}$ The transition maps are the obvious ones.

For every action of a group $H$ on a set $S$, we will denote by $[S / H]$ the associated transformation groupoid. Hence,

$$
\operatorname{DGM}\left(L^{\bullet}, A\right)=\left[\operatorname{Obj} D G M\left(L^{\bullet}, A\right) / \exp \left(L^{0} \otimes \mathfrak{m}\right)\right] .
$$

If $H$ acts on another set $Y$, we define ${ }^{4}[S / H] \bowtie Y:=[S \times Y / H]$.

Remark 2.1. In the notation of [Man99], $M C_{L^{\bullet}}(A)=\operatorname{Obj} D G M\left(L^{\bullet}, A\right)$ and $D e f_{L^{\bullet}}=$ Iso $\operatorname{DGM}\left(L^{\bullet},-\right)$.

\footnotetext{
1 We are working in the category of sets enriched with a functorial version of all small limits and colimits.

2 See [GoMi88, p. 52] for this notion.

3 See [GoMi88, 2.2, p. 53]. The action is defined in 1.3 there, pp. 50-51, using the BakerCampbell-Hausdorff formula. The differentiable structure on $\exp \left(L^{0} \otimes \mathfrak{m}\right)$ will not be used, only its set-theoretic group structure.

4 See [GoMi88, p. 63]. This could also be viewed as a homotopy fiber product $[S / H] \bowtie Y=$ $[S / H] \times[* / H][Y / H]$.
} 
Proposition 2.2. If $H^{0}\left(L^{\bullet}\right)=0$ then Iso $\operatorname{DGM}\left(L^{\bullet},{ }_{-}\right)$is prorepresentable. In general, for every splitting $\delta$ of $L^{\bullet}$, a hull in the sense of [Sch69], $\operatorname{Kur}_{L^{\bullet}} \cdot \rightarrow \operatorname{Def}_{L^{\bullet}}$, can be constructed; it is called the formal Kuranishi space.

Proof. See [GoMi90], and also [Man99].

For the reader's convenience, we recall:

Definition 2.3. Let $F$ be a covariant functor of Artin rings. Let $T$ be the formal spectrum of a complete local algebra $R$ and $\phi: T \rightarrow F$ a morphism of functors ${ }^{5}$ given by $\xi \in$ $F(T)$. Then $\phi$ is a hull if it has the following properties:

(1) $\phi\left(\mathbb{C}[\epsilon] /\left(\epsilon^{2}\right)\right)$ is an isomorphism.

(2) For every Artin ring $B$ and $w \in F(B)$ there is a morphism $\psi: R \rightarrow B$ such that $w=\psi(\xi)$.

If unicity occurs in the last property then $F$ is prorepresentable by its hull.

2.1.2. The Deligne-Goldman-Millson groupoid attached to an augmented dgla. Let $\mathfrak{g}$ be a Lie algebra over $\mathbb{C}$, viewed as a dgla in degree 0 , and let $\epsilon: L^{\bullet} \rightarrow \mathfrak{g}$ be an augmentation of the dgla $L^{\bullet}$. Let $(A, \mathfrak{m})$ be an Artin local $\mathbb{C}$-algebra. Then one defines the small groupoid $\operatorname{DGM}\left(L^{\bullet}, \epsilon, A\right)$ by:

$$
\begin{aligned}
\operatorname{Obj} D G M\left(L^{\bullet}, \epsilon, A\right) & =\left\{\left(\alpha, e^{r}\right) \in L^{1} \otimes \mathfrak{m} \times \exp (\mathfrak{g} \otimes \mathfrak{m}) \mid d \alpha+\frac{1}{2}[\alpha, \alpha]=0\right\}, \\
\operatorname{Hom}\left(\left(\alpha, e^{r}\right),\left(\beta, e^{s}\right)\right) & =\left\{\lambda \in L^{0} \otimes \mathfrak{m} \mid \exp (\lambda) \alpha=\beta, \exp (\epsilon(\lambda)) \cdot e^{r}=e^{s}\right\} .
\end{aligned}
$$

In the preceding notation, one has

$$
\operatorname{DGM}\left(L^{\bullet}, \epsilon, A\right)=\operatorname{DGM}\left(L^{\bullet}, A\right) \bowtie \exp (\mathfrak{g} \otimes \mathfrak{m})_{\epsilon},
$$

the $\epsilon$ subscript meaning that the gauge group acts via $\epsilon$.

This gives a covariant functor $\operatorname{DGM}\left(L^{\bullet}, \epsilon,-\right)$ on the category Art of $\mathbb{C}$-Artin local rings with values in the category of small groupoids.

Proposition 2.4. If $\epsilon: H^{0}\left(L^{\bullet}\right) \rightarrow \mathfrak{g}$ is injective then $\operatorname{DGM}\left(L^{\bullet}, \epsilon,-\right)$ is a discrete functor in groupoids, then Def $L_{L^{\bullet}, \epsilon}=\operatorname{Iso} \operatorname{DGM}\left(L^{\bullet}, \epsilon,-\right)$ is prorepresentable. For every splitting $\delta$ of $\left(L^{\bullet}, \epsilon\right)$ one can construct an explicit formal scheme and an isomorphism $\operatorname{Kur}_{L \cdot, \epsilon}^{\delta} \rightarrow \operatorname{Def}_{L \cdot, \epsilon}$.

Remark 2.5. $\operatorname{Kur}_{L^{\bullet}, \epsilon}^{\delta} \rightarrow \operatorname{Def}_{L^{\bullet}, \epsilon}$ is uniquely determined up to a unique isomorphism.

Proof. The proof, implicit in [GoMi88], is an adaptation of [GoMi90] with an $\epsilon$. It may nevertheless be useful to give an outline.

Suppose $\left(K^{\bullet}, d,[],\right)$ is a positively graded dgla, $\mathfrak{h}$ a Lie algebra, and $\epsilon: K^{0} \rightarrow \mathfrak{h}$ a Lie algebra map. Suppose we are given decreasing filtrations $M^{\bullet} K^{\bullet}$ and $M^{\bullet} \mathfrak{g}$ such that $\left[M^{i}, M^{j}\right] \subset M^{i+j}$ and $d, \epsilon: M^{i} \rightarrow M^{i}$. Assume the nilpotency condition $K^{\bullet}=M^{1} K^{\bullet}$ and $\mathfrak{h}=M^{1} \mathfrak{h}$, and $M^{k}=0$ for $k \gg 0$. In this case the Lie brackets are nilpotent.

\footnotetext{
5 We use the Yoneda lemma to justify using the same notation for $T$ and its functor of points.
} 
A splitting is a collection of maps denoted $\delta: K^{i} \rightarrow K^{i-1}$ such that $\delta^{2}=0, d=d \delta d$ and $\delta=\delta d \delta$. In this case, we get a decomposition

$$
K^{i}=\operatorname{im}(d) \oplus \operatorname{im}(\delta) \oplus(\operatorname{ker}(d) \cap \operatorname{ker}(\delta)) .
$$

Indeed, any element $u$ can be written as $u=d \delta(u)+\delta d(u)+(1-d \delta-\delta d)(u)$ and any decomposition $u=d u_{1}+\delta u_{2}+u_{3}$ with $d u_{3}=0, \delta u_{3}=0$ has to be of that form. Conversely, suppose we are given a decomposition $K^{i}=A^{i} \oplus B^{i} \oplus C^{i}$ such that $d\left(K^{i-1}\right)=A^{i}$ and $\operatorname{ker}(d)=A^{i} \oplus C^{i}$. Then $d: B^{i-1} \stackrel{\cong}{\rightarrow} A^{i}$ and we can define $\left.\delta\right|_{A^{i}}$ to be the inverse, extended by 0 on $B^{i}$ and $C^{i}$. The above decomposition associated to $\delta$ is the same as the given $A^{i} \oplus B^{i} \oplus C^{i}$. So, to give a splitting is the same thing as to give such a decomposition.

We also assume thet there is given a splitting $\delta: \mathfrak{h} \rightarrow H^{0}\left(K^{\bullet}\right)$ such that $\epsilon(u)=$ $\epsilon \delta \epsilon(u)$ for any $u \in H^{0}\left(K^{\bullet}\right)=\operatorname{ker}\left(d: K^{0} \rightarrow K^{1}\right)$. This is equivalent to specifying a subspace $\operatorname{ker}(\delta) \subset \mathfrak{h}$ complementary to $\epsilon\left(H^{0}\left(K^{\bullet}\right)\right)$. Injectivity of $\epsilon: H^{0}\left(K^{\bullet}\right) \rightarrow \mathfrak{h}$ then implies that $\delta(v)=\delta \epsilon \delta(v)$ for any $v \in \mathfrak{h}$.

We assume that our differentials and splittings strictly preserve the filtration $M^{\bullet}$. This is equivalent to requiring the direct sum decomposition to be compatible with the filtration in the sense that the filtered vector space $K^{i}$ is the direct sum of the subspaces with their induced filtrations (and the same for $\mathfrak{h}$ ).

With the above notation, recall the gauge fixing procedure underlying GoldmanMillson's construction [GoMi90]:

Lemma 2.6. Suppose $\eta \in K^{1}$ with $d(\eta)+\frac{1}{2}[\eta, \eta]=0$, and $r \in \mathfrak{h}$. Then there is a unique gauge transformation $e^{s}$ for $s \in K^{0}$, taking $\left(\eta, e^{r}\right)$ to a new Maurer-Cartan element $\left(\zeta, e^{z}\right)$ such that $\delta(\zeta)=0$ and $\delta(z)=0$.

Proof. The proof is by induction on the filtration $M^{\bullet}$. Suppose we have chosen our gauge transformation to get to $\left(\zeta, e^{z}\right)$ with $\delta(\zeta) \in M^{k} K^{0}$ and $\delta(z) \in M^{k} H^{0}\left(K^{\bullet}\right)$. Then use the gauge transformation $s=-\delta(\zeta)-\delta(z)$. Modulo $M^{k+1}$ the effect of this gauge transformation adds $d(s)=-d \delta(\zeta)$ to $\zeta$, and adds $\epsilon(s)=-\epsilon \delta(z)$ to $z$. But $\delta(\zeta-d \delta(\zeta))=0$ and $\delta(z-\epsilon \delta(z))=0$, so the new element $\left(\zeta_{1}, e^{z_{1}}\right)$ has $\delta(\zeta) \in M^{k+1} K^{0}$ and $\delta(z) \in M^{k+1} H^{0}\left(K^{\bullet}\right)$. This proves the existence of the gauge transformation in question. Uniqueness is proved similarly: if $\left(\eta, e^{r}\right)$ already satisfies the gauge-fixing property and $e^{s}$ is a gauge transformation taking it to another $\left(\zeta, e^{z}\right)$ with this property, then we can prove by induction that $s \in M^{k} K^{0}$ for all $k$, so $s=0$.

Let us turn to the construction of the representing formal scheme for the functor $A \mapsto$ Iso $\operatorname{DGM}(L, \epsilon ; A)$ for the proof of Proposition 2.4. Choose splittings as described previously, for the dgla $L^{\bullet}$. In the analytic case, one possible choice of $\delta$ is to compose $d^{*}$ with the Green's operator. Note that $\delta$ will not be in any way compatible with the Lie bracket. However, it induces morphisms also denoted by $\delta$ on the complexes $L \otimes \mathfrak{m}_{A}$ for any artinian algebra $A$. We apply the gauge-fixing procedure to the dgla $K^{\bullet}:=L^{\bullet} \otimes \mathfrak{m}_{A}$ with filtration $M^{k} K^{\bullet}:=L^{\bullet} \otimes \mathfrak{m}_{A}^{k}$. This filtration will be compatible with the splitting $\delta$ and satisfies the required nilpotency condition. 
Similarly choose a map $\delta: \mathfrak{g} \rightarrow H^{0}(L)$ which again induces a splitting on $\mathfrak{h}:=$ $\mathfrak{g} \otimes \mathfrak{m}_{A}$, which is compatible with the filtration $M^{k} \mathfrak{h}:=\mathfrak{g} \otimes \mathfrak{m}_{A}^{k}$.

By Lemma 2.6 applied with $K^{\bullet}=L^{\bullet} \otimes \mathfrak{m}$ we have $\mathfrak{h}=\mathfrak{g} \otimes \mathfrak{m}$, any Maurer-Cartan element $\left(\eta, e^{r}\right)$ is gauge-equivalent to a unique Maurer-Cartan element $\left(\zeta, e^{z}\right)$ such that $\delta(\zeta)=0$ and $\delta(z)=0$ in addition to the Maurer-Cartan equation $d(\zeta)+\frac{1}{2}[\zeta, \zeta]=0$. By hypothesis $(*)$ the gauge transformation is unique. In this way we obtain a functor

$$
\begin{aligned}
& A \mapsto D G M^{\delta}(L, \epsilon ; A) \\
& \quad:=\left\{\left(\zeta, e^{r}\right) \mid r \in \mathfrak{g} \otimes \mathfrak{m}_{A}, \zeta \in L^{1} \otimes \mathfrak{m}_{A}, d(\zeta)+\frac{1}{2}[\zeta, \zeta]=0, \delta(\zeta)=0, \delta(r)=0\right\} .
\end{aligned}
$$

This is given by algebraic equations but in a possibly infinite-dimensional space $L^{1}$. We can reduce to a finite-dimensional space in the following way. Let $H^{1}:=\operatorname{ker}(d) \cap$ $\operatorname{ker}(\delta) \subset L^{1}$. We can solve the equations

$$
\delta d(\zeta)+\frac{1}{2} \delta[\zeta, \zeta]=0 \quad \operatorname{in} \operatorname{im}\left(\delta: L^{2} \rightarrow L^{1}\right)
$$

because $\delta d$ maps $L^{1}$ surjectively onto $\operatorname{im}(\delta)$. The space of solutions is maped isomorphically to $H^{1}$ by the projection $P^{1}: L^{1} \rightarrow H^{1}$ which vanishes on $\operatorname{im}(d)+\operatorname{im}(\delta)$. Hence the functor of formal solutions in an artinian local algebra $A$ is represented by the formal completion of the vector space $H^{1}$ at the origin. Let $\widehat{H}^{1} \times \widehat{\Omega}$ denote the space of solutions $\left(\zeta, e^{r}\right)$ with $\delta(r)=0$. Let $P^{2}: L^{2} \rightarrow H^{2}$ be the projection vanishing on the images of $d$ and $\delta$. Then the map $\zeta \mapsto P^{2}([\zeta, \zeta])$ is a formal regular function from $\widehat{H}^{1}$ to $H^{2}$, and $D G M^{\delta}(L, \epsilon)$ is the zero-set of this map. This gives an explicit representation of the functor and finishes the proof of Proposition 2.4.

\subsection{Goldman-Millson isomorphisms}

\subsubsection{Deforming representations of a Kähler group. Let $x \in X$ and}

$$
\epsilon_{x}: H^{\bullet}\left(X, \operatorname{End}\left(\mathbb{V}_{\rho}\right)\right) \rightarrow \operatorname{End}\left(\mathbb{V}_{\rho}\right)_{x}
$$

be the augmentation of the Hodge dgla $H^{\bullet}=H^{\bullet}\left(X, \operatorname{End}\left(\mathbb{V}_{\rho}\right)\right)$ by evaluation at $x$. We also define, for every Artin local algebra $A$, a group $G_{A}^{0}=\operatorname{ker}\left(G L\left(\mathbb{V}_{\rho, x}\right)(A) \rightarrow\right.$ $\left.G L\left(\mathbb{V}_{\rho, x}\right)\right){ }^{6}$ Then we have a morphism of groups $\exp \left(H^{0} \otimes \mathfrak{m}\right) \rightarrow G_{A}^{0}$ induced by the augmentation map. The left action gives a set-theoretic action of $\exp \left(H^{0} \otimes \mathfrak{m}\right)$ on $G_{A}^{0}$, which can be used to define the transformation groupoid

$$
D G M\left(H^{\bullet}, A\right) \bowtie G_{A}^{0}=\left[\operatorname{Obj} D G M\left(L^{\bullet}, A\right) \times G_{A}^{0} / \exp \left(L^{0} \otimes \mathfrak{m}\right)\right] .
$$

Note also that $G_{A}^{0}=\exp \left(\operatorname{End}\left(\mathbb{V}_{\rho}\right)_{x} \otimes \mathfrak{m}\right)$ so

$$
\operatorname{DGM}\left(H^{\bullet}, A\right) \bowtie G_{A}^{0}=\operatorname{DGM}\left(H^{\bullet}, \epsilon_{x}, A\right)
$$

in the notation of 2.1.2.

\footnotetext{
${ }^{6}$ See [GoMi88, 3.9 p. 66]; $G_{-}^{0}$ is a prorepresentable group object in the category of covariant set-valued functors on $\underline{\text { Art. }}$
} 
Proposition 2.7. The functor $h_{G M}$ on $\underline{\text { Art defined by }}$

$$
A \mapsto \operatorname{Iso} D G M\left(H^{\bullet}, A\right) \bowtie G_{A}^{0}
$$

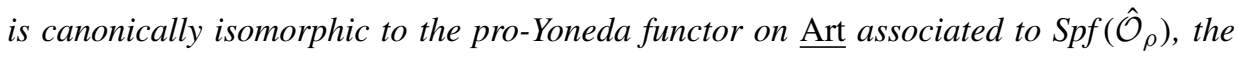
germ at $\rho$ of $R\left(\Gamma, G L\left(\mathbb{V}_{\rho, x}\right)\right)$, i.e. to the functor $h_{R\left(\Gamma, G L\left(\mathbb{V}_{\rho, x}\right)\right)_{\rho}}$ :

$$
\left(A \mapsto R\left(\Gamma, G L\left(\mathbb{V}_{\rho, x}\right)\right)(A)_{\rho}=\left\{\rho_{A} \in R\left(\Gamma, G L\left(\mathbb{V}_{\rho, x}\right)\right)(A) \mid \rho_{A} \bmod \mathfrak{m}=\rho\right\}\right) .
$$

Proof. This is actually what is proven in [GoMi88]. The combination of Theorem 3.5 p. 63 , Theorem 6.8 p. 82 and the argument in Sections 7-8 there implies that the first functor prorepresents $\operatorname{Spf}\left(\hat{\mathcal{O}}_{\rho}\right)$. But the isomorphism of functors constructed in [GoMi88] is actually canonically defined. Since we want to be as explicit as possible, we shall give a more detailed sketch of their argument.

Let $M^{\bullet} \subset E^{\bullet}$ be the subdgla of $D^{c}$-closed twisted forms inside the dgla of Lemma 1.11. Then the natural projection $M^{i} \rightarrow H_{D^{c}}^{i}\left(X, \operatorname{End}\left(\mathbb{V}_{\rho}\right)\right)$ and the Hodge-theoretic canonical isomorphism $H_{D^{c}}^{i}\left(X, \operatorname{End}\left(\mathbb{V}_{\rho}\right)\right) \rightarrow H^{i}\left(X, \operatorname{End}\left(\mathbb{V}_{\rho}\right)\right)$ gives rise to a dgla morphism from $M^{\bullet}$ to $H^{\bullet}$. Now, the $D^{\prime} D^{\prime \prime}$-lemma implies (see [GoMi88, Sect. 7] or [D-S75]) that

$$
E^{\bullet} \leftarrow M^{\bullet} \rightarrow H^{\bullet}
$$

is a dgla quasiisomorphism. Using the $\operatorname{DGM}(-,-)$ functor and [GoMi88, Corollary 2.12 p. 59], we get a diagram of objectwise equivalences of functors from $\underline{\text { Art }}$ to Gpd:

$$
\operatorname{DGM}\left(E^{\bullet},-\right) \leftarrow \operatorname{DGM}\left(M^{\bullet},-\right) \rightarrow \operatorname{DGM}\left(H^{\bullet},-\right) .
$$

Since the above quasiisomorphism commutes with the natural augmentation $\epsilon_{x}: E^{\bullet} \rightarrow$ $\operatorname{End}\left(\mathbb{V}_{\rho}\right)_{x}$, we deduce ${ }^{7}$ equivalences of functors in groupoids:

$$
\operatorname{DGM}\left(E^{\bullet},-\right) \bowtie G_{-}^{0} \leftarrow \operatorname{DGM}\left(M^{\bullet},-\right) \bowtie G_{-}^{0} \rightarrow \operatorname{DGM}\left(H^{\bullet},-\right) \bowtie G_{-}^{0} .
$$

Now, an object $\alpha$ of $\operatorname{DGM}\left(E^{\bullet}, A\right)$ can be interpreted as a flat $A$-linear connection $D_{\alpha}$ on $C^{\infty}\left(\mathbb{V}_{\rho} \otimes A\right)$ such that $D_{\alpha}=D_{\mathbb{V}_{\rho}} \otimes \operatorname{Id}_{A} \bmod \mathfrak{m}$. Then we see that holonomy defines a map from $\operatorname{Obj} \operatorname{DGM}\left(E^{\bullet}, A\right)$ to $R\left(\Gamma, G L\left(\mathbb{V}_{\rho, x}\right)\right)(A)_{\rho}$. [GoMi88, Sect. 6] states (implicitly) that this can be enhanced to a natural equivalence of groupoids

$$
\operatorname{DGM}\left(E^{\bullet}, A\right) \rightarrow\left[R\left(\Gamma, G L\left(\mathbb{V}_{\rho, x}\right)\right)(A)_{\rho} / G_{A}^{0}\right] .
$$

Eliminating the superfluous isotropy using identity as a basepoint for $G_{A}^{0}$ gives a natural equivalence of groupoids:

$$
\left[R\left(\Gamma, G L\left(\mathbb{V}_{\rho, x}\right)\right)(A)_{\rho} / G_{A}^{0}\right] \bowtie G_{A}^{0} \leftarrow\left[R\left(\Gamma, G L\left(\mathbb{V}_{\rho, x}\right)\right)(A)_{\rho} /\{\operatorname{Id}\}\right]
$$

Since we have a natural identification

$$
\operatorname{Iso}\left[R\left(\Gamma, G L\left(\mathbb{V}_{\rho, x}\right)\right)(A)_{\rho} /\{\operatorname{Id}\}\right]=R\left(\Gamma, G L\left(\mathbb{V}_{\rho, x}\right)\right)(A)_{\rho},
$$

\footnotetext{
7 See [GoMi88, Sections 3.7-3.9 pp. 63-64].
} 
passing to Iso in the above long chain of objectwise natural equivalences gives the required natural isomorphism.

The above proposition gives a different proof of the representability of Proposition 2.4 in the special cases $L^{\bullet}=E^{\bullet}, H^{\bullet}$ or $M^{\bullet}$. That was in fact the original proof of representability in [GoMi88].

Corollary 2.8. Let $\underline{\operatorname{Art}}_{n}$ be the full subcategory of $\underline{\operatorname{Art}}$ whose objects $(A, \mathfrak{m})$ satisfy $\mathfrak{m}^{n+1}$ $=0$. Then the Yoneda functor on $\underline{\mathrm{Art}}_{n}$ corepresented by $\mathcal{O}_{\rho \mid n}$ is canonically isomorphic to the restriction to $\underline{\operatorname{Art}}_{n}$ of

$$
A \mapsto \operatorname{Iso} D G M\left(H^{\bullet}, A\right) \bowtie G_{A}^{0} .
$$

2.2.2. Parameters for Goldman-Millson isomorphisms. Let $\Omega_{H_{\rho}^{1}}^{0}$ be the formal germ at 0 of End $\left(\mathbb{V}_{\rho}\right)_{x} / \epsilon\left(H^{0}\left(X, \operatorname{End}\left(\mathbb{V}_{\rho}\right)\right)\right)$ and let $\left(S_{1}, \mathfrak{m}_{1}\right)$ be its complete local algebra. Let $Q_{H_{\rho}^{1}}$ be the formal germ at 0 of the quadratic cone

$$
\left\{\alpha \in H^{1}\left(X, \operatorname{End}\left(\mathbb{V}_{\rho}\right)\right) \mid[\alpha, \alpha]=0\right\},
$$

and $\left(S_{2}, \mathfrak{m}_{2}\right)$ be its complete local algebra.

Let $h_{1}$ and $h_{2}$ be the pro-Yoneda functors of $\Omega_{H_{\rho}^{1}}^{0}$ and $Q_{H_{\rho}^{1}}$. Note that $S_{1}$ (resp. $S_{2}$ ) prorepresents $h_{1}$ (resp. $h_{2}$ ).

Definition 2.9. A formal subscheme $t \subset\left(\operatorname{End}\left(\mathbb{V}_{\rho}\right)_{x}, 0\right)$ transverse to $\epsilon\left(H^{0}\right)$ will be called a GM-transversal.

A GM-transversal $\mathfrak{t}$ is smooth or equivalently its complete local ring is isomorphic to a ring of power series. In fact, we will tacitly assume that the transversals we consider are attached to a linear subspace, although more general choices could be envisioned.

Theorem 2.10. To every GM-transversal $\mathfrak{t}$, we can associate a well defined isomorphism of covariant functors on Art,

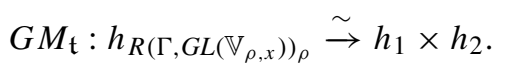

With this choice, the inclusion of $h_{1}$ in $h_{R\left(\Gamma, G L\left(\mathbb{V}_{\rho, x}\right)\right)_{\rho}}$ is the transformation of functors induced by the inclusion of the formal germ at $\rho$ of $\Omega_{\rho}$ into the formal germ $R\left(\Gamma, G L\left(\mathbb{V}_{\rho, x}\right)\right)_{\rho}$.

Proof. In [GoMi88, Lemma 3.10], an isomorphism of functors between $h_{G M}$ and $h_{1} \times h_{2}$ is constructed. By definition:

$$
\begin{aligned}
h_{1}(A) & \left.=\operatorname{End}\left(\mathbb{V}_{\rho}\right)_{x} \otimes \mathfrak{m} / \epsilon\left(H^{0}\left(X, \operatorname{End}\left(\mathbb{V}_{\rho}\right)\right)\right) \otimes \mathfrak{m}\right), \\
h_{2}(A) & =\operatorname{Obj} D G M\left(H^{\bullet}, A\right), \\
h_{G M}(A) & =h_{2}(A) \times \exp \left(\operatorname{End}\left(\mathbb{V}_{\rho}\right)_{x} \otimes \mathfrak{m}\right) / \exp \left(\epsilon\left(H^{0}\left(X, \operatorname{End}\left(\mathbb{V}_{\rho}\right)\right)\right) \otimes \mathfrak{m}\right) .
\end{aligned}
$$


Although this point is not made explicitly in [GoMi88], one way to define precisely the isomorphism is to prescribe the additional choice of a formal subscheme $\mathfrak{t} \subset\left(\operatorname{End}\left(\mathbb{V}_{\rho}\right)_{x}, 0\right)$ transverse to $\epsilon\left(H^{0}\right)$. The exponential gives an isomorphism $h_{\mathfrak{t}} \rightarrow h_{1}$ and a natural isomorphism $i: h_{\mathfrak{t}} \times h_{2} \rightarrow h_{G M}$ by

$$
i\left(t, \eta_{2}\right)=\left(\eta_{2}, \exp (t)\right) / \exp \left(\epsilon\left(H^{0}\left(X, \operatorname{End}\left(\mathbb{V}_{\rho}\right)\right) \otimes \mathfrak{m}\right)\right)
$$

This choice of a Goldman-Millson isomorphism has indeed the required property, by inspection of the construction.

Since $\epsilon\left(H^{0}\right)$ is actually a sub-Hodge structure of the polarized Hodge structure $\operatorname{End}\left(\mathbb{V}_{\rho}\right)_{x}$, the germ $\mathfrak{t}_{H}$ of the orthogonal complement is a canonical choice for $\mathfrak{t}$; we will call this choice the Hodge transversal. Admittedly, this is a rather artificial way of rigidifying Goldman-Millson's theorem. Hence, it seems abusive to call the resulting isomorphism canonical and we will call $G M_{\mathfrak{t}_{H}}^{c}$ the preferred Goldman-Millson isomorphism.

Definition 2.11. The isomorphism $G M_{\mathfrak{t}_{H}}^{c}: R\left(\Gamma, G L\left(\mathbb{V}_{\rho, x}\right)\right)_{\rho} \rightarrow \Omega_{H_{\rho}^{1}}^{0} \times T$ constructed above will be called the preferred Goldman-Millson isomorphism attached to the $\mathbb{C}$ VHS $\rho$.

In a less artificial way, we have:

Remark 2.12. The morphisms $h_{2} \rightarrow h_{G M}$ and $h_{G M} \rightarrow h_{1}$ are independent of the choice

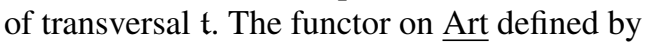

$$
h_{1}^{\prime}: A \mapsto \exp \left(\operatorname{End}\left(\mathbb{V}_{\rho, x}\right) \otimes \mathfrak{m}\right) / \exp \left(\epsilon\left(H^{0}\left(X, \operatorname{End}\left(\mathbb{V}_{\rho}\right)\right) \otimes \mathfrak{m}\right)\right)
$$

is isomorphic to $h_{1}$ and the projection map $h_{G M} \rightarrow h_{1}^{\prime}$ is a retraction of the inclusion $h_{1}^{\prime} \rightarrow h_{G M}$ given by $A \mapsto\left(0, h_{1}^{\prime}(A)\right) / \exp \left(\epsilon\left(H^{0}\left(X, \operatorname{End}\left(\mathbb{V}_{\rho}\right)\right) \otimes \mathfrak{m}\right)\right)$.

Let us restate this in terms of complete local algebras.

Corollary 2.13. Choose a GM-transversal t. There is an isomorphism $i_{\mathfrak{t}}^{c}: \hat{\mathcal{O}}_{\rho} \rightarrow S_{1} \hat{\otimes} S_{2}$. The ideal $\mathfrak{j}$ is mapped by this isomorphism to $S_{1} \hat{\otimes} \mathfrak{m}_{2}$. Passing to the quotient, it induces an isomorphism $\mathcal{O}_{\rho \mid n} \rightarrow S_{1} \hat{\otimes} S_{2} / \mathfrak{m}^{n}$, which will also be denoted by $i_{\mathfrak{t}}^{c}$.

Proof. This follows from the previous theorem, and the construction of [Sch69].

We define the ideal $\mathfrak{q}$ as the ideal mapped to $\mathfrak{m}_{1} \hat{\otimes} S_{2}$ by the dual of the preferred GoldmanMillson isomorphism. The formal subscheme $T=\operatorname{Spf}\left(\hat{\mathcal{O}}_{T}\right) \subset \operatorname{Spf}\left(\hat{\mathcal{O}}_{\rho}\right)$ defined by $q$ will be called the preferred formal slice at $\rho$. Note that $\hat{\mathcal{O}}_{T}$ is canonically isomorphic to $S_{2}$, and in fact the formal subscheme $T$, representing the functor $h_{2}$, is embedded in $h_{G M}$ independently of the choice of the transversal $t$. The adic filtration $\left(j^{n}\right)_{n \in \mathbb{N}}$ induces on the complete local ring $\hat{\mathcal{O}}_{T}=\left(\hat{\mathcal{O}}_{\rho} / \mathfrak{q}, \mathfrak{m}\right)$ the filtration $\left(\mathfrak{m}^{n}\right)_{n \in \mathbb{N}}$. 
2.2.3. Interpretation in terms of formal Kuranashi space. Since the dgla with a trivial differential, $\left(H_{D^{c}}^{\bullet}\left(X, \operatorname{End}\left(\mathbb{V}_{\rho}\right)\right), 0\right)$, has just one splitting, namely $\delta=0, T$ is isomorphic through a uniquely defined tautological isomorphism to the uniquely defined Kuranishi formal space of $H_{D^{c}}^{\bullet}\left(X, \operatorname{End}\left(\mathbb{V}_{\rho}\right)\right)$.

If we fix a Kähler metric on $X$, Hodge theory enables us to define the orthogonal splitting of the dgla $E^{\bullet}=E^{\bullet}\left(X, \operatorname{End}\left(\mathbb{V}_{\rho}\right)\right)$. The preceding chain of quasiisomorphisms gives rise to a natural map $\kappa^{c}: T \rightarrow \operatorname{Def}_{E} \bullet$ which is a hull.

Holonomy at $x \in X$ gives a canonical isomorphism $h_{x}: \operatorname{Def} E_{E} \rightarrow \operatorname{Def}(\rho)$ where $\operatorname{Def}(\rho)$ is the Yoneda functor of Artin rings germ at $\rho$ of the groupoid $R\left(\Gamma, G L\left(\mathbb{V}_{\rho, x}\right)\right) / /$ $G L\left(\mathbb{V}_{\rho, x}\right)$ attached to the conjugation action.

Hence $h_{x}^{c}: h_{x} \circ \kappa^{c}: T \rightarrow \operatorname{Def}(\rho)$ is just a hull of the deformation functor $\operatorname{Def}(\rho)$. In particular for every $g \in \operatorname{Aut}(T / \operatorname{Def}(\rho)), h_{x} \circ \kappa \circ g$ is another hull. One easily establishes, using [Rim72] (or [Rim80]):

Lemma 2.14. $\operatorname{Aut}(T / \operatorname{Def}(\rho)) \subset \operatorname{ker}\left(\operatorname{Aut}\left(\hat{\mathcal{O}}_{T}, \mathfrak{m}\right) \rightarrow \operatorname{Aut}\left(\hat{\mathcal{O}}_{T} / \mathfrak{m}^{2}\right)\right)$ is the image of the natural map $\exp \left(H^{0}\left(X, \operatorname{End}\left(\mathbb{V}_{\rho}\right)\right) \otimes \mathfrak{m}^{2}\right) \rightarrow \operatorname{Aut}(T)$.

Observe that $H^{0}\left(X, \operatorname{End}\left(\mathbb{V}_{\rho}\right)\right)$ is nothing but the Lie algebra of the reductive algebraic group $H \subset G L\left(\mathbb{V}_{\rho, x}\right)$ which centralizes $\rho$. The group $H$ consists of the automorphisms of the local system $\mathbb{V}_{\rho}$, hence it acts on $T$. On the other hand, choosing a vector space complementary to $\epsilon\left(H^{0}\left(X, \operatorname{End}\left(\mathbb{V}_{\rho}\right)\right)\right)$ in $\mathfrak{g l}\left(\mathbb{V}_{\rho, x}\right)$ (call the resulting linear transversal $\mathfrak{t}$ ) is tantamount to choosing a splitting of the cohomology augmented Lie algebra. Hence the choice of a linear transversal gives us a uniquely defined natural isomorphism of punctual formal schemes

$$
\mathcal{G} \mathcal{M}^{c}: G L\left(\widehat{\left.\mathbb{V}_{\rho, x}\right)} \times_{H} T_{\rho} \rightarrow R\left(\Gamma, G L\left(\mathbb{V}_{\rho, x}\right)\right)_{\rho}=\operatorname{Spf}\left(\hat{\mathcal{O}}_{\rho}\right) .\right.
$$

In general, the best basepoint free way to state the main result of [GoMi88] is that there is a canonical equivalence of formal groupoids

$$
[T / H] \rightarrow\left[R\left(\Gamma, G L\left(\mathbb{V}_{\rho, x}\right)\right) / G L\left(\mathbb{V}_{\rho, x}\right)\right]_{\rho}
$$

where the second groupoid is the germ at $\rho$ of the conjugation groupoid. This is actually independent of $x$ (or more precisely, independent up to a unique isomorphism).

\subsection{Split $\mathbb{C}-M H S$ on $\hat{\mathcal{O}}_{\rho}$ and $\hat{\mathcal{O}}_{T}$}

Since $H^{1}\left(X, \operatorname{End}\left(\mathbb{V}_{\rho}\right)\right)$ is a weight one $\mathbb{C}$-HS, $H^{2}\left(X, \operatorname{End}\left(\mathbb{V}_{\rho}\right)\right)$ is a weight two $\mathbb{C}$-HS, and

$$
p=[-,-]: S^{2} H^{1}\left(X, \operatorname{End}\left(\mathbb{V}_{\rho}\right)\right) \rightarrow H^{2}\left(X, \operatorname{End}\left(\mathbb{V}_{\rho}\right)\right)
$$

respects the Hodge structure, we deduce that $H^{1}\left(X, \operatorname{End}\left(\mathbb{V}_{\rho}\right)\right)^{*}$ is a $\mathbb{C}$-HS of weight -1 and

$$
I_{2}=\operatorname{Im}\left(p^{t}: H^{2}\left(X, \operatorname{End}\left(\mathbb{V}_{\rho}\right)\right)^{*} \rightarrow S^{2} H^{1}\left(X, \operatorname{End}\left(\mathbb{V}_{\rho}\right)\right)^{*}\right)
$$

is a weight $-2 \mathbb{C}$-HS. Hence

$$
I_{n}=I_{2} S^{n-2} H^{1}\left(X, \operatorname{End}\left(\mathbb{V}_{\rho}\right)\right)^{*} \subset S^{n} H^{1}\left(X, \operatorname{End}\left(\mathbb{V}_{\rho}\right)\right)^{*}
$$


is a weight $-n$ sub- $\mathbb{C}$-Hodge structure. Hence,

$$
\Pi_{n}=S^{n} H^{1}\left(X, \operatorname{End}\left(\mathbb{V}_{\rho}\right)\right)^{*} / I_{n}
$$

is a weight $-n \mathbb{C}$-HS and the algebra $S_{2}^{H}:=\sum_{n \in \mathbb{N}} \Pi_{n}$ viewed as an infinite-dimensional split $\mathbb{C}$-MHS with an algebra structure respecting the MHS. The weight filtration is exactly $W_{-n} S_{2}^{H}=\mathfrak{m}_{2}^{n}$ since $\mathfrak{m}_{2}=\sum_{n>0} \Pi_{n}$ is a maximal ideal of the complete local algebra $S_{2}^{H}$, and $\Pi_{1}$ generates $\mathfrak{m}_{2}$.

The formerly introduced complete local algebra $S_{2}$ is canonically isomorphic to the algebra obtained from $S_{2}^{H}$ by forgetting the Hodge filtrations, hence $\hat{\mathcal{O}}_{T}$ carries a canonical split $\mathbb{C}$-MHS whose weight filtration is given by the powers of the maximal ideal.

On the other hand $S_{1}$ is the free complete local algebra generated by the weight zero HS $\left(\operatorname{End}\left(\mathbb{V}_{\rho}\right) / \epsilon\left(H^{0}\left(X, \operatorname{End}\left(\mathbb{V}_{\rho}\right)\right)\right)\right)^{*}$. It can thus be viewed as a weight zero $\mathbb{C}$-Hodge algebra.

Hence $S_{1} \hat{\otimes} S_{2}$ carries a canonical split $\mathbb{C}$-MHS whose weight filtration is given by the powers of $S_{1} \hat{\otimes} m_{2}$.

Proposition 2.15. The filtration $W_{\bullet}$ is the weight filtration of a split $\mathbb{C}$-mixed Hodge structure on $\mathcal{O}_{\rho \mid n}$. Passing to the limit we get a MHS on $\hat{\mathcal{O}}_{\rho}$.

Proof. Choose a GM-transversal $\mathfrak{t}$. Using the isomorphism $G M_{\mathfrak{t}}$, we transfer this mixed Hodge structure to $\hat{\mathcal{O}}_{\rho}$, the weight filtration being defined by the powers of $\mathfrak{j}$. The power $\mathfrak{m}^{n}$ of the maximal ideal is easily seen to correspond to the split sub- $\mathbb{C}$-MHS $\sum_{k+l>n} S^{k} H^{0}\left(X, \operatorname{End}\left(\mathbb{V}_{\rho}\right)\right) \otimes \Pi_{l}$. Thus, the powers of $\mathfrak{j}$ are the weight filtration of a $\mathbb{C}$-MHS on $\mathcal{O}_{\rho \mid n}$. This concludes the proof.

\section{Mixed-Hodge-theoretical aspects of Goldman-Millson's theory at a $\mathbb{C}$-VHS}

This section continues our exegesis of [GoMi88] and aims at making more explicit the use of formality. Despite the fact that the preferred Goldman-Millson isomorphism is rather artificial, $T$ does not depend on any choices, so an explicit description should be available.

An explicit construction of three 'canonical' hulls will be described next. They will not coincide in general. One of them corresponds to the preferred Goldman-Millson isomorphism constructed above.

The two new slices will be used in the last sections to produce the $\mathbb{C}$-MHS and $\mathbb{C}$ VMHS we are seeking.

\subsection{Preliminary remarks and definitions}

3.1.1. Universal Maurer-Cartan elements. Assume $T=\operatorname{Spf}\left(O_{T}\right)$ is a formal scheme and $L^{\bullet}$ is a dgla. In order to define the map $h: T \rightarrow D e f_{L}$. for a hull, one needs to construct a universal Maurer-Cartan element for this hull, i.e. an element of $\operatorname{Obj} D G M\left(L^{\bullet}, O_{T}\right)$ whose gauge equivalence class gives rise to $h$. 
Definition 3.1. Assume $T=\operatorname{Spf}\left(O_{T}\right)$ is a formal scheme and $L^{\bullet}$ is a dgla. A universal Maurer-Cartan element for $L^{\bullet}$ defined on $T$ is an element of $\operatorname{Obj} \operatorname{DGM}\left(L^{\bullet}, O_{T}\right)$ whose gauge equivalence class gives rise to a hull.

Observe that the hull $\mathrm{Kur}_{L}^{\delta}$ • canonically attached to a splitting $\delta$ as in Proposition 2.2 carries a tautological universal Maurer-Cartan element.

The following lemma restates the versality property (2) of Definition 2.3.

Lemma 3.2. Let $\eta \in \operatorname{Obj} D G M\left(L^{\bullet}, T\right)$ be a universal Maurer-Cartan element and suppose $\eta^{\prime} \in \operatorname{Obj} D G M\left(L^{\bullet}, S\right)$ is another Maurer-Cartan element. Then there exists a ring morphism $\phi: T \rightarrow S$ and a gauge transformation $e^{r} \in \exp \left(L^{0} \otimes \mathfrak{m}_{S}\right)$ such that $\eta^{\prime}=e^{r} . \phi(\eta)$. Unicity for $(\phi, r)$ holds if $H^{0}\left(L^{\bullet}\right)=0$.

If $T$ is an object of $\underline{\operatorname{Art}}_{n}$ (i.e. satisfies $\mathfrak{m}_{T}^{n+1}=0$ ) and $(T, \eta)$ satisfies the preceding universal property with respect to $\underline{\mathrm{Art}}_{n}$ then we say that $(T, \eta)$ is a universal MaurerCartan element of order $n$.

3.1.2. Choice of a model. The construction of $\mathcal{G} \mathcal{M}^{c}$ depends on the choice of $\left(\operatorname{ker}\left(D^{c}\right), D\right)$ setting up a quasiisomorphism between $E^{\bullet}\left(X, \operatorname{End}\left(\mathbb{V}_{\rho}\right)\right)$ and its cohomology algebra. This is fine if $\rho$ is actually real for a certain real structure since $\mathcal{G} \mathcal{M}^{c}$ will be defined over the reals. The real Zariski closure of the monodromy group being Hodge, it carries a real structure, but it is not clear how the real structure extends in the non-Zariski dense case. If we drop the reality constraint, other very natural choices are available.

We may indeed choose $\left(\operatorname{ker}\left(D^{\prime}\right), D\right)$ and $\left(\operatorname{ker}\left(D^{\prime \prime}\right), D\right)$ as intermediate models resulting in two alternative formal Kuranishi spaces $h_{x}^{\prime}: T \rightarrow \operatorname{Def}(\rho)$ and $h_{x}^{\prime \prime}: T \rightarrow \operatorname{Def}(\rho)$.

\subsection{Constructing a $\mathbb{C}-V M H S$}

In this section we first construct a universal Maurer-Cartan element for $T$ using $\left(\operatorname{ker}\left(D^{\prime}\right), D\right)$, then another one using $\left(\operatorname{ker}\left(D^{\prime \prime}\right), D\right)$, and glue them together.

\subsubsection{The $D^{\prime} D^{\prime \prime}$-lemma}

Lemma 3.3. Let $\eta_{1}, \ldots, \eta_{b} \in E^{\bullet}$ form a basis of the subspace $\mathcal{H}^{1}\left(X\right.$, End $\left.\left(\mathbb{V}_{\rho}\right)\right)$ of harmonic twisted one-forms, each $\eta_{i}$ being of pure Hodge type $\left(P_{i}, Q_{i}\right)$ for the DeligneZucker $\mathbb{C}$-MHC structure on $E^{\bullet}$. Then $\left\{\eta_{i}\right\}$ is a basis of $H^{1}\left(X, \operatorname{End}\left(\mathbb{V}_{\rho}\right)\right)$ whose dual basis for $\Pi_{1}=H^{1}\left(X, \operatorname{End}\left(\mathbb{V}_{\rho}\right)\right)^{*}$ is denoted by $\left(\left\{\eta_{1}\right\}^{*}, \ldots,\left\{\eta_{b}^{*}\right\}\right)$. The $\operatorname{End}\left(\mathbb{V}_{\rho}\right) \otimes \Pi_{1}$ valued one-form $\alpha_{1}$ defined by

$$
\alpha_{1}:=\sum_{i=1}^{b} \eta_{i} \otimes\left\{\eta_{i}\right\}^{*}
$$

is D-closed and $D^{c}$-closed.

Proof. This is a consequence of the already mentioned Deligne-Zucker construction [Zu79].

Let $\pi_{2}: \Pi_{1} \otimes \Pi_{1} \rightarrow \Pi_{2}$ be the product mapping constructed above (see ( $\dagger$ ) in $\S 2.3$ ). 
Lemma 3.4. $\beta_{2}=\pi_{2}\left(\left[\alpha_{1}, \alpha_{1}\right]\right)$ is D-closed, $D^{c}$-closed and cohomologous to 0 .

Proof. $\left[\alpha_{1}, \alpha_{1}\right] \in E^{2} \otimes \Pi_{1} \otimes \Pi_{1}$ is $D^{\prime}$ - and $D^{\prime \prime}$-closed since $E^{\bullet}$ is a dgla and $D^{\prime}$ also preserves the Lie bracket. $\pi_{2}$ being a linear map, $\beta_{2}$ is $D^{\prime}$ - and $D^{\prime \prime}$-closed.

Let $\phi: \Pi_{2} \rightarrow \mathbb{C}$ be a linear form. Then $\phi$ can be interpreted as an element $\sum_{1 \leq i, j \leq b} c_{i, j}\left\{\eta_{i}\right\} \otimes\left\{\eta_{j}\right\}$ in the kernel of

$$
[-,-]: \otimes^{2} H^{1}\left(X, \operatorname{End}\left(\mathbb{V}_{\rho}\right)\right) \rightarrow H^{2}\left(X, \operatorname{End}\left(\mathbb{V}_{\rho}\right)\right)
$$

i.e. such that

$$
\sum_{1 \leq i, j \leq b} c_{i, j}\left[\left\{\eta_{i}\right\},\left\{\eta_{j}\right\}\right]=0 .
$$

Hence $\phi\left(\beta_{2}\right)=\sum_{1 \leq i, j \leq b} c_{i, j} \eta_{i} \wedge \eta_{j}$. If $\left(c_{i, j}\right)$ is any antisymmetric matrix, then this form vanishes and $(R)$ holds. If $\left(c_{i, j}\right)$ is symmetric and satisfies $(R)$ then

$$
\phi\left(\beta_{2}\right)=\frac{1}{2} \sum_{i, j} c_{i, j}\left[\eta_{i}, \eta_{j}\right] .
$$

Since $\left[\eta_{i}, \eta_{j}\right]$ is a de Rham representative of $\left[\left\{\eta_{i}\right\},\left\{\eta_{j}\right\}\right], \phi\left(\beta_{2}\right)$ is cohomologous to zero thanks to $(R)$. Hence $\beta_{2}$ is cohomologous to zero.

Lemma 3.5. There is a form $\gamma_{2} \in E^{0} \otimes \Pi_{2}$ such that $D^{\prime} D^{\prime \prime} \gamma_{2}=\beta_{2}$. Define $\alpha_{2} \in E^{1} \otimes \Pi_{2}$ to be $\frac{1}{2} D^{\prime} \gamma_{2}$. Then $\alpha_{2}$ is $D^{\prime}$-exact and satisfies $D \alpha_{2}+\frac{1}{2} \beta_{2}=0$.

Proof. This is a consequence of the $D^{\prime} D^{\prime \prime}$-lemma: see for instance [D-S75].

If $\alpha \in E^{1} \otimes \Pi_{b}$ and $\beta \in E^{1} \otimes \Pi_{b}$ we define $\alpha \wedge \beta \in E^{2} \otimes \Pi_{a+b}$ using composition of matrices and product in $\Pi$. With this notation $\alpha_{1} \wedge \alpha_{1}=\frac{1}{2} \beta_{2}$.

Lemma 3.6. $\beta_{3}=\alpha_{1} \wedge \alpha_{2}+\alpha_{2} \alpha_{1}$ is $D^{\prime}$-exact and $D^{\prime \prime}$-closed. By the $D^{\prime} D^{\prime \prime}$-lemma we conclude $\beta_{3}=D^{\prime} D^{\prime \prime} \gamma_{3}$ and we define $\alpha_{3}=D^{\prime} \gamma_{3}$.

Proof. $D \alpha_{2}+\alpha_{1} \alpha_{1}=0$. Hence $D \beta_{3}=\alpha_{1} \wedge \alpha_{1} \wedge \alpha_{1}-\alpha_{1} \wedge \alpha_{1} \wedge \alpha_{1}$.

Lemma 3.7. For $k \geq 3$ we can construct a $D^{\prime}$-exact form $\alpha_{k} \in E^{1} \otimes \Pi_{k}$ such that

$$
D \alpha_{k}+\alpha_{k-1} \alpha_{1}+\alpha_{k-2} \alpha_{2}+\cdots+\alpha_{1} \alpha_{k-1}=0 .
$$

Proof. We have done the $k=3$ case explicitly. The first step for $k \rightarrow k+1$ is to observe that $\alpha_{k} \alpha_{1}+\cdots+\alpha_{1} \alpha_{k}$ is $D^{\prime \prime}$-closed and $D^{\prime}$-exact and then apply the $D^{\prime} D^{\prime \prime}$-lemma.

Lemma 3.8. $\sum_{k=1}^{\infty} \alpha_{k}$ is a universal Maurer-Cartan element for $T$ and $\left(\operatorname{ker}\left(D^{\prime}\right), D\right)$.

Proof. This follows from the form of $\alpha_{1}$.

Remark 3.9. Since $\mathcal{H}^{1}\left(X, \operatorname{End}\left(\mathbb{V}_{\rho}\right)\right)=\operatorname{ker}\left(D^{\prime}: E^{1} \rightarrow E^{2}\right) \cap \operatorname{ker}\left(D^{\prime \prime}: E^{1} \rightarrow E^{2}\right)$ and $\operatorname{ker}\left(D^{\prime} D^{\prime \prime}: E^{0} \rightarrow E^{2}\right)=\operatorname{ker}\left(D^{\prime \prime}\right)$ the construction of $\left(\alpha_{k}\right)_{k \geq 1}$ is canonical, purely complex analytic (does not depend on the Kähler structure), functorial and the $\alpha_{k}$ are uniquely determined. 
3.2.2. A canonical connection. Let $\left(V_{n}, D\right)$ be the smooth vector bundle with a flat connection underlying the local system

$$
\mathbb{V}_{\rho} \otimes_{\mathbb{C}}\left(\hat{\mathcal{O}}_{T} / \mathfrak{m}^{n}\right)=\bigoplus_{k=-n}^{0} \mathbb{V}_{\rho} \otimes \Pi_{-k}
$$

The weight filtration on $\left(\hat{\mathcal{O}}_{T} / \mathfrak{m}^{n}\right)$ gives rise to a filtration $\left\{\left(W_{k} V_{n}, D\right)\right\}_{-n \leq k \leq 0}$. Actually $\mathbb{V}_{\rho} \otimes_{\mathbb{C}}\left(\hat{\mathcal{O}}_{T} / \mathfrak{m}^{n}\right)$ is naturally a split VMHS, whose monodromy representation is just a direct sum of copies of the initial representation $\rho$.

This is not the local system we are interested in for Theorem 1-rather, it is the associated-graded-so we should perturb it by adding a new term to the connection. Let $A_{k}^{\prime}: V_{n} \rightarrow V_{n} \otimes \mathcal{E}_{X}^{1}$ be defined as $\sum_{l} A_{k}^{l}$ where $A_{k}^{l}: \mathbb{V}_{\rho} \otimes \Pi_{l} \rightarrow \mathbb{V}_{\rho} \otimes \Pi_{l+k} \otimes \mathcal{E}_{X}^{1}$ is the natural multiplication by $\alpha_{k}$.

Proposition 3.10. $D+\sum_{k=1}^{n} A_{k}^{\prime}$ is a flat $\left(\hat{\mathcal{O}}_{T} / \mathfrak{m}^{n}\right)$-linear connection on the filtered smooth vector bundle $\left\{\left(W_{k} V_{n}\right)\right\}_{-n \leq k \leq 0}$. Its holonomy representation $\rho_{T, n}: \pi_{1}(X, x) \rightarrow$ $G L\left(\mathbb{V}_{\rho, x} \otimes\left(\hat{\mathcal{O}}_{T} / \mathfrak{m}^{n}\right)\right)$ passes to the limits and gives rise to a representation $\rho_{T}: \pi_{1}(X, x)$ $\rightarrow G L\left(\mathbb{V}_{\rho, x} \otimes \hat{\mathcal{O}}_{T}\right)$ which induces the Goldman-Millson slice $T \rightarrow R\left(\Gamma, G L\left(\mathbb{V}_{\rho, x}\right)\right)$.

Proof. The fact that the connection is flat is a restatement of the construction in the previous subsection. The link with the construction in [GoMi88] can be easily made by inspecting that reference.

3.2.3. Nontriviality of the extension. Already at $n=2$ we can see that the new representation $\rho_{T, 2}$ will not be semisimple. Indeed, the associated local system $\mathbb{V}_{\rho, T, 2}$ is an extension

$$
0 \rightarrow \mathbb{V}_{\rho} \otimes\left(\mathfrak{m} / \mathfrak{m}^{2}\right) \rightarrow \mathbb{V}_{\rho, T, 2} \rightarrow \mathbb{V}_{\rho} \rightarrow 0
$$

whose extension class is the element of

$$
H^{1}\left(\operatorname{Hom}\left(\mathbb{V}_{\rho}, \mathbb{V}_{\rho} \otimes\left(\mathfrak{m} / \mathfrak{m}^{2}\right)\right)\right)=H^{1}\left(\operatorname{End}\left(\mathbb{V}_{\rho}\right)\right) \otimes \mathfrak{m} / \mathfrak{m}^{2}
$$

corresponding to the identity matrix under the identification

$$
\mathfrak{m} / \mathfrak{m}^{2} \cong H^{1}\left(\operatorname{End}\left(\mathbb{V}_{\rho}\right)\right)^{*} .
$$

So, as soon as $\operatorname{dim}\left(\mathfrak{m} / \mathfrak{m}^{2}\right)>0$ the extension is nontrivial.

3.2.4. Griffiths transversality. Since $\mathbb{V}_{\rho} \otimes \Pi_{-k}$ carries a weight $-k \mathbb{C}$-HS, $\left(V_{n}, D\right)$ underlies a split VMHS whose Hodge and anti-Hodge bundles are:

$$
\mathcal{F}^{p}\left(V_{n}\right)=\bigoplus_{k=-n}^{0} \mathcal{F}^{p}\left(\mathbb{V}_{\rho} \otimes \Pi_{-k}\right), \quad \overline{\mathcal{G}}^{q}\left(V_{n}\right)=\bigoplus_{k=-n}^{0} \overline{\mathcal{G}}^{q}\left(\mathbb{V}_{\rho} \otimes \Pi_{-k}\right)
$$


Lemma 3.11. The connection $D+A^{\prime}=D+\sum_{k=1}^{n} A_{k}^{\prime}$ is Griffiths transversal in the sense that

$$
\begin{aligned}
& \left(D+\sum_{k=1}^{n} A_{k}^{\prime}\right)^{1,0} C^{\infty}\left(\mathcal{F}^{q}\right) \subset C^{\infty}\left(\mathcal{F}^{q-1}\right) \otimes \Omega^{1,0}, \\
& \left(D+\sum_{k=1}^{n} A_{k}^{\prime}\right)^{0,1} C^{\infty}\left(\mathcal{F}^{q}\right) \subset C^{\infty}\left(\mathcal{F}^{q}\right) \otimes \Omega^{0,1}
\end{aligned}
$$

Proof. Observe that the twisted one-form $\alpha_{1}$ is a Hodge type $(0,0)$ vector in a weight zero HS. Since it is in $\mathcal{G}^{0}$ it follows that $A_{1}^{\prime} . \mathcal{F}^{q}\left(V_{n}\right) \subset \mathcal{F}^{q}\left(V_{n} \otimes E^{1}\right)$, i.e.

$$
A_{1}^{\prime} \cdot \mathcal{F}^{q}\left(V_{n}\right) \subset \mathcal{F}^{q} \otimes \Omega^{0,1}+\mathcal{F}^{q-1} \otimes \Omega^{1,0} .
$$

Now, $\beta_{2}$ is also a Hodge type $(0,0)$ vector in a weight $0 \mathrm{HS}$. Hence, $\gamma_{2}$ is also a Hodge type $(-1,-1)$ vector in a weight -2 HS. Hence $\alpha_{2}$ is of Hodge type $(0,-1)$ in the weight $-1 \mathrm{HS} E^{1} \otimes \Pi_{2}$. Hence $\alpha_{2} \in \mathcal{F}^{0} \cap \overline{\mathcal{G}}^{-1}\left(E^{1} \otimes \Pi_{2}\right)$. Since it is in $\mathcal{F}^{0}$ it follows that $A_{2}^{\prime} \cdot \mathcal{F}^{q}\left(V_{n}\right) \subset \mathcal{F}^{q}\left(V_{n} \otimes E^{1}\right)$.

Continuing this way, we see that $\alpha_{k}$ is of Hodge type $(0,1-k)$ and

$$
\left(\sum A_{k}^{\prime}\right) \cdot \mathcal{F}^{q}\left(V_{n}\right) \subset \mathcal{F}^{q}\left(V_{n} \otimes E^{1}\right) .
$$

This is Griffiths transversality.

\subsubsection{The second filtration}

Remark 3.12. $D+\left(\sum_{k=1}^{n} A_{k}^{\prime}\right)$ is not Griffiths antitransversal for the above definition of $\overline{\mathcal{G}}^{\bullet}$.

So, we need to introduce the following variant of our basic construction:

Lemma 3.13. Let $\alpha_{1}^{v}$ denote the twisted one-form $\alpha_{1}$ called by another name. For $k \geq 2$, we can construct a $D^{\prime \prime}$-exact form $\alpha_{k}^{v} \in E^{1} \otimes \Pi_{k}$ such that

$$
D^{\prime} \alpha_{k}^{v}+\alpha_{k-1}^{v} \alpha_{1}^{v}+\alpha_{k-2}^{v} \alpha_{2}^{v}+\cdots+\alpha_{1}^{v} \alpha_{k-1}^{v}=0
$$

$\alpha_{k}^{v}$ is of Hodge type $(1-k, 0)$ and $D+A^{\prime \prime}=D+\sum \alpha_{k}^{v}$ satisfies Griffiths antitransversality for the above $\overline{\mathcal{G}}^{\bullet}$.

In order to get a VMHS structure on $\rho_{T, n}$ one needs to prove the following proposition where $\mathfrak{m}$ stands for the maximal ideal in $\left(\hat{\mathcal{O}}_{T} / \mathfrak{m}^{n}\right)$ :

Proposition 3.14. There is a $W_{\bullet}$-preserving gauge transformation $g$ and $\phi^{*} \in$ $\operatorname{Aut}\left(\left(\hat{\mathcal{O}}_{T} / \mathfrak{m}^{n}\right)\right)$ such that:

(1) $g \in \exp \left(E^{0} \otimes \mathfrak{m}^{2}\right)$, hence induces $\operatorname{Id}$ on $G r_{W}^{\bullet}\left(V_{n}\right)$,

(2) $\left(\phi^{*}: \mathfrak{m} / \mathfrak{m}^{2} \rightarrow \mathfrak{m} / \mathfrak{m}^{2}\right)=\mathrm{Id}_{\mathfrak{m} / \mathfrak{m}^{2}}$, hence $\operatorname{Id}_{\mathbb{V}_{\rho}} \otimes \phi^{*}$ preserves $W_{\bullet}$ and induces $\mathrm{Id}$ on $G r_{W}^{\bullet}\left(V_{n}\right)$

(3) $D+A^{\prime \prime}=g\left(\operatorname{Id}_{\mathbb{V}_{\rho}} \otimes \phi^{*}\right)\left(D+A^{\prime}\right)\left(\operatorname{Id}_{\mathbb{V}_{\rho}} \otimes \phi^{*}\right)^{-1} g^{-1}$. 
Proof. Let us remark first that the automorphisms of $\left(\hat{\mathcal{O}}_{T} / \mathfrak{m}^{n}\right)$ act on the group of gauge transformations $\exp \left(E^{0} \otimes \mathfrak{m}\right)$ so that the group of filtered bundle automorphisms they generate is isomorphic to the semidirect product deduced from this action.

Since $A_{1}^{\prime}=A_{1}$, the statement is obviously true for $n=1$. Since $\alpha_{2}^{v}=+\frac{1}{2} D^{\prime} \gamma_{2}$, we have $\alpha_{2}^{v}-\alpha_{2}=D \gamma_{2}$. Then $\exp \left( \pm \gamma_{2}\right)$ is the required gauge transformation for $n=2$, in which case we can still set $\phi^{*}=$ Id. For larger $n$, we have not been able to get such an explicit construction of the pair $\left(g, \phi^{*}\right)$.

In general, since $A^{\prime}$ and $A^{\prime \prime}$ come from a universal Maurer-Cartan element, this is a consequence of the universality property given in Lemma 3.2.

Theorem 3.15. The filtered vector bundle $\left(V_{n}, W_{\bullet}\right)$ with connection $D+A^{\prime}$ constructed in Proposition 3.10, Hodge filtration constructed in Lemma 3.11, and anti-Hodge filtration defined by transporting the anti-Hodge filtration constructed in Lemma 3.11 using the bundle automorphism $g\left(\operatorname{Id}_{V_{\rho}} \otimes \phi^{*}\right)$ constructed in Proposition 3.14 gives rise to a $\mathbb{C}$-VMHS whose holonomy is the filtered representation $\rho_{T, n}$.

Proof. Since the bundle automorphism contsructed in Proposition 3.14 induces the identity on $G r_{W}^{\bullet}\left(V_{n}\right)$, the new Hodge filtration still defines on each stalk a $\mathbb{C}$-VMHS thanks to Lemma 1.5. Griffiths antitransversality is obtained by transport of structure from Lemma 3.13.

This concludes the proof of Theorem 1 .

Remark 3.16. In the real case, if one insists on using the definition that $\overline{\mathcal{F} \bullet}$ is the complex conjugate of $\mathcal{F}^{\bullet}$, one has to transport $\mathcal{F}^{\bullet}$ to a real model, for instance the model given by $\left(\operatorname{ker} D^{c}, D\right)$ using a real fundamental automorphism couple and a pair of conjugate gauge transformations.

Remark 3.17. In case $H^{0}\left(X, \operatorname{End}\left(\mathbb{V}_{\rho}\right)\right) \neq \mathbb{C} I d$, this VMHS is not uniquely defined, since we may twist it by the action of the ambiguity group.

\section{3. $\mathbb{C}-M H S$ on $\hat{\mathcal{O}}_{\rho}$}

We may use the preceding construction and Lemma 1.5 to put a $\mathbb{C}$-MHS on $\hat{\mathcal{O}}_{T}$ which will be only defined up to the action of the ambiguity group $\operatorname{Aut}(T / \operatorname{Def}(\rho))$ constructed in Lemma 2.14.

Since the reductive group $H$ acts as a group of automorphisms of $\mathbb{V}_{\rho}$, the whole construction is $H$-invariant [Rim80], which yields a uniquely defined $\mathbb{C}$-MHS on $O_{T}^{H}$ which is the ring of formal series at $[\rho]$ in $M_{B}\left(X, G L\left(\mathbb{V}_{\rho, x}\right)\right)$.

To treat the case of $\mathcal{O}_{\rho \mid n}$, we need to introduce an augmented version of the preceding construction.

3.3.1. Filtered Goldman-Millson theory. Consider the following situation: we are given a nonnegatively graded dgla $L$ with a decreasing filtration $G^{\bullet}$ such that $d: G^{p} L \rightarrow G^{p} L$ and [, ] : $G^{p} L \times G^{q} L \rightarrow G^{p+q} L$. Let $G r_{G}(L)=\bigoplus G^{p} L / G^{p+1} L$ be the associatedgraded dgla. Suppose furthermore that we are given a finite-dimensional Lie algebra $\mathfrak{g}$ 
and an augmentation $\epsilon: L^{0} \rightarrow \mathfrak{g}$ compatible with the Lie bracket. Suppose $\mathfrak{g}$ is also given a filtration denoted $G^{\bullet} \mathfrak{g}$, compatible with the Lie bracket, and $\epsilon$ is compatible with the filtrations.

Suppose $B$ is an artinian local algebra also provided with a decreasing filtration denoted $G^{\bullet} B$ compatible with the algebra structure $G^{p} B \times G^{q} A \rightarrow G^{p+q} A$, with $1_{B} \in G^{0} B$. We assume that the filtration is exhaustive, that is, $G^{p} B=B$ for $p \ll 0$ and $G^{p} B=0$ for $p \gg 0$. Let $\mathfrak{m}_{B}$ denote the maximal ideal of $B$, which has its induced filtration $G^{p} \mathfrak{m}_{B}:=G^{p} B \cap \mathfrak{m}_{B}$.

Let $\exp \left(\mathfrak{g} \otimes \mathfrak{m}_{B}\right)$ denote the nilpotent Lie group associated to the nilpotent Lie algebra $\mathfrak{g} \otimes \mathfrak{m}_{B}$

An augmented Maurer-Cartan element is a pair $\left(\eta, e^{r}\right)$ with

$$
\eta \in L^{1} \otimes_{\mathbb{C}} \mathfrak{m}_{B}, \quad d(\eta)+\frac{1}{2}[\eta, \eta]=0,
$$

and $e^{r} \in \exp \left(\mathfrak{g} \otimes \mathfrak{m}_{B}\right)$. Let $\operatorname{Obj} \operatorname{DGM}(L, \epsilon ; B)$ denote the space of augmented MaurerCartan elements. An element $\left(\eta, e^{r}\right)$ is compatible with the filtrations if $\eta \in G^{0}\left(L^{1} \otimes \mathfrak{m}_{B}\right)$ and $\beta \in \exp \left(G^{0}\left(\mathfrak{g} \otimes \mathfrak{m}_{B}\right)\right)$. Let $\operatorname{Obj} \operatorname{DGM}(L, \epsilon ; B)^{G}$ denote the subspace of elements compatible with the filtration.

The group $\exp \left(L^{0} \otimes \mathfrak{m}_{B}\right)$, with elements denoted $e^{s}$, acts as a group of gauge transformations which acts on both components.

The group $\exp \left(G^{0}\left(L^{0} \otimes \mathfrak{m}_{B}\right)\right)$ acts on $\operatorname{Obj} D G M(L, \epsilon ; B)^{G}$. Let

$$
\operatorname{DGM}(L, \epsilon ; B)^{G}:=\left[\operatorname{Obj} D G M(L, \epsilon ; B)^{G} / \exp \left(G^{0}\left(L^{0} \otimes \mathfrak{m}_{B}\right)\right)\right]
$$

denote the filtered Deligne-Goldman-Millson groupoid, the quotient groupoid of the filtered-compatible space by the filtered gauge group.

Suppose $K$ is another dgla, with filtration $G^{\bullet} K$ and an augmentation $\varepsilon$ towards the same $\mathfrak{g}$. A filtered augmented quasiisomorphism from $K$ to $L$ is a morphism of dgla's $\psi: K \rightarrow L$, compatible with the filtrations, making a commutative square with the augmentations, and such that $G_{G}(\psi): G r_{G}(K) \rightarrow G r_{G}(L)$ is a quasiisomorphism.

The quasiisomorphism invariance of the Deligne-Goldman-Millson groupoid generalizes here:

Proposition 3.18. Suppose $\psi$ is a filtered augmented quasiisomorphism. Then the induced map

$$
\operatorname{DGM}(K, \epsilon ; B)^{G} \stackrel{D G M\left(\psi ; 1_{B}\right)}{\longrightarrow} \operatorname{DGM}(L, \epsilon ; B)^{G}
$$

is an equivalence of groupoids.

Proof. Let $M^{\bullet}$ be the filtration of $\mathfrak{m}_{B}$ by powers of $\mathfrak{m}_{B}$. We can choose a common splitting $\mathfrak{m}_{B}=\bigoplus V^{p, q}$ for the two filtrations $M^{\bullet}$ and $G^{\bullet}$ on the vector space $\mathfrak{m}_{B}$, not necessarily compatible with the algebra structure. Thus $M^{k}=\bigoplus_{p \geq k} V^{p, q}$ and $G^{r}=$ $\bigoplus_{q \geq r} V^{p, q}$. The filtration $M^{\bullet}$ induces filtrations going by the same name on $K^{\bullet} \otimes \mathfrak{m}_{B}$ 
and $L^{\bullet} \otimes \mathfrak{m}_{B}$. Furthermore, we can express the filtrations $G^{\bullet}$ on these complexes as

$$
\begin{aligned}
G^{r}\left(L^{\bullet} \otimes \mathfrak{m}_{B}\right) & =\bigoplus_{p, j} G^{j}\left(L^{\bullet}\right) \otimes V^{p, r-j}, \\
G^{r}\left(K^{\bullet} \otimes \mathfrak{m}_{B}\right) & =\bigoplus_{p, j} G^{j}\left(K^{\bullet}\right) \otimes V^{p, r-j} .
\end{aligned}
$$

These expressions also give splittings for the filtrations $M^{\bullet}$. These expressions are compatible with the differential, but not with the bracket. However, the condition for a map to be a filtered quasiisomorphism depends only on the differential. Thus, the morphism $K^{\bullet} \otimes \mathfrak{m}_{B} \rightarrow L^{\bullet} \otimes \mathfrak{m}_{B}$ is a bifiltered quasiisomorphism with respect to the pair of filtrations $G^{\bullet}, M^{\bullet}$. In particular, the morphism

$$
G^{0}\left(K^{\bullet} \otimes \mathfrak{m}_{B}\right) \rightarrow G^{0}\left(L^{\bullet} \otimes \mathfrak{m}_{B}\right)
$$

is a filtered quasiisomorphism for the filtration $M^{\bullet}$, and this filtration makes the bracket nilpotent (that is, the bracket is trivial on the associated graded of $M^{\bullet}$ ). A similar discussion holds for the filtrations on $\mathfrak{g} \otimes \mathfrak{m}_{B}$.

The groupoid in the filtered case $D G M(K, \epsilon ; B)^{G}$ is just the groupoid of MaurerCartan elements in $G^{0}\left(K^{\bullet} \otimes \mathfrak{m}_{B}\right)$ together with a framing in $G^{0}\left(\mathfrak{g} \otimes \mathfrak{m}_{B}\right)$, and the same for $L^{\bullet}$. Using [GoMi88, Cor. 2.12 p. 59], we conclude that

$$
D G M(K, \epsilon ; B)^{G} \stackrel{D G M\left(\psi ; 1_{B}\right)}{\longrightarrow} D G M(L, \epsilon ; B)^{G}
$$

is an equivalence of groupoids.

On the other hand, if $f: B \rightarrow B^{\prime}$ is a morphism of filtered artinian local algebras, we obtain a morphism

$$
\operatorname{DGM}(L, \epsilon ; B)^{G} \stackrel{D G M\left(1_{L} ; f\right)}{\longrightarrow} D G M\left(L, \epsilon ; B^{\prime}\right)^{G} .
$$

Say that an element $\left(\eta, e^{r}\right) \in \operatorname{Obj} D G M(L, \epsilon ; B)^{G}$ is an order $k$ universal augmented filtered Maurer-Cartan element if $\mathfrak{m}_{B}^{k}=0$ and, for any artinian local algebra with an exhaustive filtration $\left(R, G^{\bullet}\right)$ such that $\mathfrak{m}_{R}^{k}=0$ the map $f \mapsto D G M\left(1_{L} ; f\right)\left(\eta, e^{r}\right)$ induces an equivalence from the discrete groupoid of filtered algebra morphisms $B \rightarrow R$ to the filtered DGM groupoid

$$
\operatorname{Hom}_{\text {filtalg }}\left(\left(B, G^{\bullet}\right),\left(R, G^{\bullet}\right)\right) \stackrel{\sim}{\rightarrow} D G M(L, \epsilon ; R)^{G} .
$$

Note in particular that this condition means that for any $R$ the objects in the groupoid $\operatorname{DGM}(L, \epsilon ; R)$ do not have nontrivial automorphisms.

Let us now develop a filtered analog of Proposition 2.4. We do not consider the general question of representability of the filtered DGM groupoid functor. Probably, in the very general case representability will not hold, so some conditions on the filtrations would be necessary. In the case of interest to us, using filtered augmented quasiisomorphisms we can reduce to a case where the filtration is decomposed, in which case it is easier to show representability. 
We say that a filtered augmented dgla $\left(L, \epsilon, G^{\bullet}\right)$ is decomposed if there exists an action of $\mathbb{C}^{*}$ on the dgla $L$ and an action on $\mathfrak{g}$ such that the augmentation is compatible with the action, and $L$ and $\mathfrak{g}$ decompose into eigenspaces for the action which split the filtrations $G^{\bullet}$. Concretely this means that we are given isomorphisms $L \cong G r_{G}(L)$ and $\mathfrak{g} \cong G r_{G}(\mathfrak{g})$ which are compatible with the differential, the bracket, and the augmentation. Denote by $L(k)$ the subspace corresponding to $G r_{G}^{k}(L)$ and similarly $\mathfrak{g}(k)$. The decomposition corresponds to the action of $\mathbb{C}^{*}$ on $(L, \mathfrak{g}, \epsilon)$.

We can use the decomposition condition to show representability of the filtered DGM groupoid functor.

Assume $\epsilon: H^{0}\left(L^{\bullet}\right) \rightarrow \mathfrak{g}$ is injective. Choose a splitting $\delta$ compatible with the decompositions of $L^{\bullet}$ and $\mathfrak{g}^{\bullet}$. We get an action of $\mathbb{C}^{*}$ on the representing formal scheme $D G M^{\delta}(L, \epsilon)$ constructed explicitly above. Let $R$ be the complete local coordinate ring of $\operatorname{Kur}_{L}^{\delta}, \epsilon$, with its universal Maurer-Cartan element $\eta$. From the construction, we see that the action on $R / \mathfrak{m}_{R}^{k}$ is an algebraic action. Furthermore, the universal Maurer-Cartan element $\eta$ is compatible with these actions of $\mathbb{C}^{*}$.

Let $G^{\bullet}$ denote the filtration of $L^{\bullet}$ and $\mathfrak{g}$ corresponding to the decomposition. Note that $d$ and $\epsilon$ are strictly compatible with $G^{\bullet}$. The decomposition of $R$ induces a filtration which we also denote by $G^{\bullet}$, and the universal Maurer-Cartan element lies in $\operatorname{DGM}(L, \epsilon ; R)^{G}$.

Theorem 3.19. Suppose $B$ is an artinian local algebra with an action of $\mathbb{C}^{*}$, and suppose $\zeta \in D G M(L, \epsilon ; B)$ is a Maurer-Cartan element fixed by $\mathbb{C}^{*}$. Then the corresponding map $v: R \rightarrow B$ and gauge transformation $w$ between $v(\eta)$ and $\zeta$, are fixed by $\mathbb{C}^{*}$.

Suppose $B$ is provided with a decreasing filtration $G^{\bullet}$ and $\left(\eta, e^{r}\right) \in \operatorname{DGM}(L, \epsilon ; B)^{G}$ is a Maurer-Cartan element compatible with the filtration. Then the map $v: R \rightarrow B$ and gauge transformation $w$ are compatible with the filtrations.

Proof. If $B$ has a decomposition and $\left(\eta, e^{r}\right)$ is a Maurer-Cartan element preserved by $\mathbb{C}^{*}$, then let $e^{s}$ be the gauge transformation going from $\left(\eta, e^{r}\right)$ to $\left(\zeta, e^{z}\right)$ with $\delta(\zeta)=0$ and $\delta(z)=0$. By unicity of $s$, we see that $s, \zeta$ and $z$ are fixed by $\mathbb{C}^{*}$. The $\left(\eta, e^{z}\right)$ give the coordinates for the map $v: R \rightarrow B$ and $s$ gives $w$, so $v$ and $w$ are fixed by $\mathbb{C}^{*}$.

Consider now the filtered case. The dgla at index 0 in the filtration can be expressed as

$$
G^{0}\left(L^{\bullet} \otimes \mathfrak{m}_{B}\right)=\bigoplus_{j} L^{\bullet}(-j) \otimes G^{j} \mathfrak{m}_{B}, \quad G^{0}\left(\mathfrak{g} \otimes \mathfrak{m}_{B}\right)=\bigoplus_{j} \mathfrak{g}(-j) \otimes G^{j} \mathfrak{m}_{B}
$$

The splitting $\delta$ is defined separately on each complex $L^{\bullet}(-j)$ and $\mathfrak{g}(-j)$, so it induces a splitting of the dgla $K^{\bullet}:=G^{0}\left(L^{\bullet} \otimes \mathfrak{m}_{B}\right)$ with Lie algebra $\mathfrak{h}:=G^{0}\left(\mathfrak{g} \otimes \mathfrak{m}_{B}\right)$. Hence the gauge fixing Lemma 2.4 can be applied to $\left(K^{\bullet}, \mathfrak{h}\right)$.

Given a Maurer-Cartan element $\left(\eta, e^{r}\right) \in \operatorname{DGM}(L, \epsilon ; B)^{G}$, this means exactly that we have a Maurer-Cartan element for $\left(K^{\bullet}, \mathfrak{h}\right)$ so by Lemma 2.6 there is a unique gauge transformation $s \in K^{0}=G^{0}\left(L^{0} \otimes \mathfrak{m}_{B}\right)$ transforming $\left(\eta, e^{r}\right)$ to a Maurer-Cartan element $\left(\zeta, e^{z}\right)$ for $\left(K^{\bullet}, \mathfrak{h}\right)$ with $\delta(\zeta)=0$ and $\delta(z)=0$. This new element is again in $\operatorname{DGM}(L, \epsilon ; B)^{G}$, and it corresponds to a morphism $v: R \rightarrow B$ which sends $R(j)$ to $G^{j}(B)$. 
Remark 3.20. When finishing this paper, we realized that the $\mathbb{C}^{*}$-invariant part of this construction was actually done in [GiKa04].

Corollary 3.21. Suppose $\left(L, \epsilon, G^{\bullet}\right)$ is a filtered augmented dgla, with finite-dimensional cohomology groups $H^{i}$ for $i=0,1,2$. Suppose that $\epsilon: H^{0} L \rightarrow \mathfrak{g}$ is injective. Suppose that $\left(L, \epsilon, G^{\bullet}\right)$ is filtered quasiisomorphic to a filtered augmented dgla which has a splitting of the filtration compatible with differential, bracket and augmentation.

Then for any $k$ there exists a filtered artinian algebra $R$ with $\mathfrak{m}_{R}^{k}=0$ and a $k$-th order universal augmented Maurer-Cartan element $\left(\eta, e^{r}\right) \in \operatorname{Obj} D G M(L, \epsilon ; R)^{G}$ compatible with the filtration. In fact, $R$ may also be split in the sense that there is an action of $\mathbb{C}^{*}$ (or equivalently $R \cong G r(B)$ ) and $\left(\eta, e^{r}\right)$ is compatible with the splitting; this splitting depends on the choice of a filtered quasiisomorphism with a split dgla.

The universal object $\left(R, G^{\bullet} ;\left(\eta, e^{r}\right)\right)$ is unique up to a unique isomorphism and gauge transformation. That is, if $\left(R^{\prime}, G^{\bullet} ;\left(\eta^{\prime}, e^{r^{\prime}}\right)\right)$ is another universal element then there is an isomorphism of filtered algebras $v:\left(R, G^{\bullet}\right) \cong\left(R^{\prime}, G^{\bullet}\right)$ and a gauge transformation $s \in \exp \left(G^{0}\left(L^{0} \otimes \mathfrak{m}_{B^{\prime}}\right)\right)$ such that $e^{s}(v(\eta))=\eta^{\prime}$. The pair $(\nu, s)$ such that $e^{s}(v(\eta))=\eta^{\prime}$ is unique.

Proof. For the split dgla, Theorem 3.19 provides the representability. By the invariance statement of Proposition 3.18, this representing object works for the original $L^{\bullet}$, and unicity comes from the universal property.

Corollary 3.22. Let $R^{\prime}$ be an artinian algebra and $\left(\eta, e^{r}\right) \in D G M\left(L^{\bullet}, \epsilon ; R\right)$ a $k$-th order universal Maurer-Cartan element. Let $\left(R, G^{\bullet}\right)$ be as in Corollary 3.21 and $\left(\eta^{\prime}, e^{r^{\prime}}\right)$ a filtered $k$-th order universal Maurer-Cartan element. Then there is a unique isomorphism $v: R^{\prime} \cong R$ and a unique gauge transformation $e^{w}$ going from $v\left(\eta^{\prime}, e^{r^{\prime}}\right)$ to $\left(\eta, e^{r}\right)$. The isomorphism $v$ induces a filtration $G^{\bullet} R^{\prime}:=v\left(G^{\bullet} R\right)$ on $R^{\prime}$.

Proof. Indeed, the splitting used to construct the universal filtered Maurer-Cartan element is a splitting that can be used to construct a universal Maurer-Cartan element, the construction being parallel. Hence the filtered Maurer-Cartan element is good enough to serve as an ordinary Maurer-Cartan element.

However, the original universal element $\left(\eta, e^{r}\right)$ is not necessarily compatible with the filtration; we have existence of a gauge transformation $e^{w}$ such that $e^{w} \cdot\left(\eta, e^{r}\right)$ is compatible with the filtration.

3.3.2. The mixed Hodge structure on the formal completion of the representation space. We now wish to apply Proposition 1.7 to the case $A=\mathcal{O}_{\rho \mid n}$. Let us first define three filtrations on this ring:

Definition 3.23. The weight filtration on $\mathcal{O}_{\rho \mid n}$ is by powers of the ideal $W_{-k}=\mathfrak{j}^{k}$. The Hodge filtration $F$ comes from Corollary 3.22 applied to the Hodge filtration of the augmented Goldman-Millson dgla $\left(E^{\bullet}\left(X, \operatorname{End}\left(\mathbb{V}_{\rho}\right)\right), \epsilon_{x}\right)$ where the augmentation is evaluation at $x$. The anti-Hodge filtration comes from the same construction. 
Theorem 3.24. These provide $A=\mathcal{O}_{\rho \mid n}$ with a $\mathbb{C}-M H S$ which is unique up to a unique isomorphism.

Proof. Let us check the conditions in Proposition 1.7.

Condition (1) comes from Goldman-Millson's theorem (indeed, the product of a quadratic cone with a vector space is again a quadratic cone).

For condition (2), note that $V$ is the dual of the space of deformations of $\rho$ in $R(X, x, G)$. This space of deformations is a relative cohomology group: $V^{*}=$ $H^{1}\left((X, x), \operatorname{End}\left(\mathbb{V}_{\rho}\right)\right)$. This has a mixed Hodge structure, which is exactly the one given by the restrictions of the filtrations $W, F, G$ above. There are only two weight quotients, in degrees 0 and -1 for $V$ or degrees 0 and 1 for $V^{*}$.

For condition (3), note that the kernel is given by the obstruction map. Since End $\left(\mathbb{V}_{\rho}\right)$ has a Lie algebra structure which is antisymmetric, the multiplication $H^{1} \times H^{1} \rightarrow H^{2}$ is symmetric, that is,

$$
\operatorname{Sym}^{2}\left(V^{*}\right)=\operatorname{Sym}^{2}\left(H^{1}\left((X, x), \operatorname{End}\left(\mathbb{V}_{\rho}\right)\right)\right) \rightarrow H^{2}\left(X, \operatorname{End}\left(\mathbb{V}_{\rho}\right)\right) .
$$

We do not include the basepoint $x$ on the right because it does not affect $H^{2}$. The transpose or dual of this map is

$$
H^{2}\left(X, \operatorname{End}\left(\mathbb{V}_{\rho}\right)\right)^{*} \rightarrow \operatorname{Sym}^{2}(V)
$$

and by Goldman-Millson's theory, the kernel $K$ as defined in the theorem is exactly the image of this map. The map is a map of $\mathbb{C}$-MHS (the target is even pure) so the image is a sub- $\mathbb{C}-$ MHS, which is condition (3).

We just have to check condition (4). For $F$, the strictness is a consequence of the fact that the Goldman-Millson formality isomorphism trivializes the Hodge filtration. In other words, the $\mathbb{C}^{*}$-action on the algebra of forms $\left(\operatorname{ker}\left(D^{\prime}\right), D^{\prime \prime}\right)$ gives a $\mathbb{C}^{*}$-action on the local ring $\mathcal{O}_{\rho \mid n}$ inducing the filtration. The map $\mu^{n}$ preserves the decomposition, so it strictly preserves the filtration.

The statement for $G$ follows from the same argument. Finally, for $W$ the statement can be seen by using the GM isomorphism $\operatorname{Spec}(A) \cong\left(\Omega_{\rho} \times T\right)_{n}$.

Remark 3.25. This $\mathbb{C}$-MHS on $\mathcal{O}_{\rho \mid n}$ is uniquely defined and only depends on the basepoint $x \in X$.

\subsection{The universal VMHS}

Theorem 3.26. The universal representation $\rho_{n}: \pi_{1}(X, x) \rightarrow G L\left(\mathbb{V}_{\rho, x} \otimes \mathcal{O}_{\rho \mid n}\right)$ is the monodromy of a Gr polarizable $\mathbb{C}$-VMHS.

Remark 3.27. This VMHS is uniquely defined and only depends on the basepoint $x \in X$.

Proof. On the bundle $\mathbb{V}_{\rho} \otimes \mathcal{O}_{\rho \mid n}$ over $X$, we have filtrations $s F, s G$ and $s W$ coming from the splitting of the Hodge decomposition on $V$, and the given filtrations on $\mathcal{O}_{\rho \mid n}$. We also have a universal Maurer-Cartan element $\left(\eta, e^{r}\right)$ where $e^{r}$ can be viewed as a framing at the point $x \in X$. However, the universal Maurer-Cartan element need not be compatible with the filtrations. Hence, there are gauge transformations $e^{f}, e^{g}$ and $e^{w}$ such 
that $e^{f} \cdot\left(\eta, e^{r}\right)$ is compatible with $s F, e^{g} \cdot\left(\eta, e^{r}\right)$ is compatible with $s G$, and $e^{w} \cdot\left(\eta, e^{r}\right)$ is compatible with $s W$. Define

$$
F:=e^{-f}(s F), \quad G:=e^{-g}(s G), \quad W:=e^{-w}(s W) .
$$

These give three filtrations on the bundle $V \otimes \mathcal{O}_{\rho \mid n}$. The connection $\nabla+\eta$ is then compatible with these, in the sense of Griffiths transversality for $F$, antitransversality for $G$, and preserves $W$ (these are because of how the filtrations $F, G$ and $W$ were defined on the algebra of forms). The action of $\mathcal{O}_{\rho \mid n}$ preserves these filtrations, and it follows from an analogue of the argument of the previous section that these filtrations define MHS's at each point; so we get a VMHS. Furthermore, the weight-graded pieces are polarizable.

We conclude this section by stating two obvious properties of the above constructions.

Lemma 3.28. The natural maps $\mathcal{O}_{\rho \mid n+1} \rightarrow \mathcal{O}_{\rho \mid n}$ are morphisms of mixed Hodge Artin local rings.

Lemma 3.29. We have a morphism of mixed Hodge rings $\mathcal{O}_{\rho \mid n} \rightarrow \operatorname{End}\left(\mathbb{V}_{\rho_{n}}\right)$.

Remark 3.30. The construction is independent of the Kähler form and functorial under morphisms $(Y, y) \rightarrow(X, x)$.

In order to see this, one has to adapt the argument of Section 3.2 starting with the obvious $\alpha_{1}$ which is the tensor corresponding to the natural map

$$
H^{1}\left((X, x), \operatorname{End}\left(\mathbb{V}_{\rho}\right)\right) \rightarrow \mathcal{H}^{1}\left(X, \operatorname{End}\left(\mathbb{V}_{\rho}\right)\right)
$$

given by composition of the Hodge isomorphism and the natural map

$$
H^{1}\left((X, x), \operatorname{End}\left(\mathbb{V}_{\rho}\right)\right) \rightarrow H^{1}\left(X, \operatorname{End}\left(\mathbb{V}_{\rho}\right)\right)
$$

\section{Representations in a reductive group $G$}

We can generalize our work until now to the case of representations in a reductive group $G$ defined over $\mathbb{C}$.

Theorem 4.1. Let $G$ be a reductive algebraic group defined over $\mathbb{C}$. Let $\sigma: \Gamma \rightarrow G(\mathbb{C})$ be a semisimple representation whose associated Higgs bundle is a fixed point of the $\mathbb{C}^{*}$-action on $M_{D o l}(X, G)$ [Sim94].

Let $R(\Gamma, G) / \mathbb{C}$ be the affine scheme parametrizing the representations of $\Gamma$ with values in $G$ endowed with the action of $G$ by conjugation. Let $\hat{\Omega}_{\sigma}$ be the formal germ at $[\sigma]$ of the orbit of $\sigma$. There is a preferred isomorphism $G M^{c}: \operatorname{Spf}\left(\hat{\mathcal{O}}_{\sigma}\right) \rightarrow \hat{\Omega}_{\sigma} \times T$ where $T$ is the formal germ at the origin of the homogeneous quadratic cone attached to the Goldman-Millson obstruction map

$$
S^{2} H^{1}\left(X, \operatorname{ad}_{\sigma}\right) \rightarrow H^{2}\left(X, \operatorname{ad}_{\sigma}\right) .
$$


Let $\hat{O}_{\sigma}$ be the complete local algebra of $R(\Gamma, G)$ at $\sigma$. Then $\hat{O}_{\sigma} / \mathfrak{m}^{n}$ carries a functorial $\mathbb{C}$-MHS whose weight filtration comes from the powers of the ideal defining the orbit of $\sigma$.

Let $\alpha$ be a rational representation of $G$ with values in $G L_{N}$ and let $\sigma_{n}: \Gamma \rightarrow$ $G\left(\hat{\mathcal{O}}_{T} / \mathfrak{m}^{n}\right)$ be the tautological representation defined in terms of $G M^{c}$. Denote by $\mathbb{V}_{\alpha, \sigma}$ the local system of $\left(\hat{\mathcal{O}}_{T} / \mathfrak{m}^{n}\right)$ free modules on $X$ attached to the representation $\alpha \circ \sigma$ : $\Gamma \rightarrow G L_{N}\left(\hat{\mathcal{O}}_{T} / \mathfrak{m}^{n}\right)$.

The $\mathbb{C}$-local system underlying $\mathbb{V}_{\alpha, \sigma}$ is the holonomy of a graded polarizable VMHS whose weight filtration is given by

$$
W_{-k} \mathbb{V}_{\alpha, \sigma}=\mathfrak{m}^{k} \cdot \mathbb{V}_{\alpha, \sigma}, \quad k=0, \ldots, n .
$$

To prove Theorem 4.1, we spell out the differences between this more general case and the case $G=G L_{n}$ treated in Theorem 1.

Let $E$ be the real Zariski closure of the monodromy group of $\sigma$. This is a real reductive subgroup of $G(\mathbb{C})$ viewed as a real reductive group. $E$ is also of Hodge type [Sim92].

Recall that a real reductive algebraic group $E$ is said to be of Hodge type if there is a morphism of real algebraic groups $h: U(1) \rightarrow \operatorname{Aut}(E)$ such that $h(-1)$ is a Cartan involution of $E$ (see [Sim92, p. 46]). By definition, $h$ is a Hodge structure on $E$. Connected groups of Hodge type are precisely those admitting an isotropic Cartan subgroup.

Consider a finite-dimensional complex representation of $E, \alpha: E \rightarrow G L\left(\mathbb{V}_{\mathbb{C}}\right)$.

Lemma 4.2. $\operatorname{ker}(\alpha)$ is fixed by $h$.

Proof. By [Sim92, p. 63, proof of Lemma 5.5] there is a morphism of real algebraic groups $z: U(1) \rightarrow E$ and an isogeny $\pi: U(1) \rightarrow U(1)$ such that $\operatorname{ad}(z)=h(\pi(z))$. Let $z^{\prime}, z \in U(1)$ be such that $\pi(z)=z^{\prime}$. Then $h\left(z^{\prime}\right)=\operatorname{ad}(z)$. Hence $h\left(z^{\prime}\right) . g \in \operatorname{ker}(\alpha)$ iff $z g z^{-1} \in \operatorname{ker}(\alpha)$ iff $g \in \operatorname{ker}(\alpha)$.

By [Sim92, Lemma 5.5], $\mathbb{V}_{\mathbb{C}}$ inherits a pure polarizable Hodge structure of weight zero and $\alpha$ is a Hodge representation in the sense of [Sim92, Lemma 5.6]. Hence, the local system attached to $\alpha \circ \sigma$ underlies a polarized $\mathbb{C}$-VHS. There is no uniqueness since the polarization is not uniquely defined and the Hodge filtration can be shifted by an integer depending on each irreducible component of $\alpha$.

The adjoint representation of $G(\mathbb{C})$ restricted to $E$ gives rise to a $\mathbb{C}$-VHS on $X$ of weight zero which we call ad ${ }_{\sigma}$. If we keep track of the real structures then we can eliminate the shift of the Hodge filtration as a source of nonuniqueness but not the polarization.

Then, we can rewrite the construction of Subsection 2.2 replacing $G L_{N}$ by $G$, using the new definition for $H^{\bullet}$ and $E^{\bullet}$ given by $H^{\bullet}=H^{\bullet}\left(X, \operatorname{ad}_{\sigma}\right)$ and $E^{\bullet}=E^{\bullet}\left(X, \operatorname{ad}_{\sigma}\right)$ and the new augmentation with values in $\mathfrak{g}$ defined by evaluation at $x$.

To adapt Subsection 2.3, we use the fact that $p=[-;-]: S^{2} H^{1}\left(X, \operatorname{ad}_{\sigma}\right) \rightarrow$ $H^{2}\left(X, \mathrm{ad}_{\sigma}\right)$ respects the Hodge structure to deduce that

$$
I_{2}=\operatorname{Im}\left(p^{t}: H^{2}\left(X, \operatorname{ad}_{\sigma}\right)^{*} \rightarrow S^{2} H^{1}\left(X, \operatorname{ad}_{\sigma}\right)^{*}\right)
$$

is a weight $-2 \mathbb{C}$-HS. Hence,

$$
I_{n}=I_{2} S^{n-2} H^{1}\left(X, \mathrm{ad}_{\sigma}\right)^{*} \subset S^{n} H^{1}\left(X, \mathrm{ad}_{\sigma}\right)^{*}
$$


is a weight $-n$ sub- $\mathbb{C}$-Hodge structure. Hence,

$$
\Pi_{n}=S^{n} H^{1}\left(X, \operatorname{ad}_{\sigma}\right)^{*} / I_{n}
$$

is a weight $-n \mathbb{C}$-HS and the algebra $S_{2}^{H}:=\sum_{n \in \mathbb{N}} \Pi_{n}$ viewed as an infinite-dimensional split $\mathbb{C}$-MHS is naturally endowed with an algebra structure respecting the MHS. The weight filtration is exactly $W_{-n} S_{2}^{H}=\mathfrak{m}_{2}^{n}$ since $\mathfrak{m}_{2}=\sum_{n>0} \Pi_{n}$ is a maximal ideal of the complete local algebra $S_{2}^{H}$, and $\Pi_{1}$ generates $\mathfrak{m}_{2}$. Thus we have $\hat{\mathcal{O}}_{\sigma} \simeq S_{2}^{H}$.

Section 3.2 is also easily adapted by using a basis of $\mathcal{H}^{1}\left(X, \mathrm{ad}_{\sigma}\right)$ in place of a basis of $\mathcal{H}^{1}\left(X\right.$, End $\left.\left(\mathbb{V}_{\rho}\right)\right)$ in Lemma 3.3 and the rest of the argument goes through without any difficulty.

In particular, we can put yet another MHS on the Artin local ring $\hat{O}_{\sigma \mid n}$ which corresponds to the $n$-th infinitesimal neighborhood of $\sigma$ and whose weight filtration is given by the powers of the ideal defining the orbit of $\sigma$. We can also interpret the universal representation $\pi_{1}(X, x) \rightarrow G\left(\hat{O}_{\sigma \mid n}\right)$ as the monodromy of a $G r$ polarizable $\mathbb{C}$-VMHS.

This concludes the proof of Theorem 4.1.

There are several natural properties of the present construction that we have not fully developed yet. For instance, the MHS on $\hat{O}_{\sigma \mid n}$ is likely to vary in a $\mathbb{C}$-VMHS when $x$ varies. We hope to understand this in a future work which should at the same time make a comparison with [Ha98].

Acknowledgments. This research is partially supported by ANR grants BLAN08-1-309225 (SEDIGA) and BLAN08-3-352054 (G-FIB).

\section{References}

[Del71] Deligne, P.: Théorie de Hodge, II. Publ. Math. IHES 40, 5-58 (1971) Zbl 0219.14007 MR 0498551

[Del75] Deligne, P.: Théorie de Hodge, III. Publ. Math. IHES 44, 6-77 (1975) Zbl 0237.14003 MR 0498552

[D-S75] Deligne, P., Griffiths, P., Morgan, J., Sullivan, D.: The real homotopy theory of Kähler manifolds. Invent. Math. 29, 245-274 (1975) Zbl 0312.55011 MR 0382702

[E-R09] Eyssidieux, P., Katzarkov, L., Pantev, T., Ramachandran, M.: Linear Shafarevich conjecture. arxiv/math:0904.0693 (2009)

[Fuj91] Fujiki, A.: Hyperkähler structure on the moduli space of flat bundles. In: Prospects in Complex Geometry (Katata, 1989), Lecture Notes in Math. 1468, Springer, 1-83 (1991) Zbl 0749.32011 MR 1123536

[GiKa04] Ginzburg, V., Kaledin, D.: Poisson deformation of symplectic quotient singularities. Adv. Math. 186, 1-57 (2004) Zbl 1062.53074 MR 2065506

[GoMi88] Goldman, W., Millson, J.: The deformation theory of representations of fundamental groups of compact Kähler manifolds. Publ. Math. IHES 67, 43-96 (1988) Zbl 0678.53059 MR 0972343

[GoMi90] Goldman, W., Millson, J.: The homotopy invariance of the Kuranishi space. Illinois J. Math. 34, 337-367 (1990) Zbl 0707.32004 MR 1046568

[Ha87] Hain, R.: The de Rham homotopy theory of complex algebraic varieties, I. K-theory 1, 271-324 (1987) Zbl 0637.55006 MR 0908993 
[Ha98] Hain, R.: The Hodge de Rham theory of relative Malcev completion. Ann. Sci. École Norm. Sup. 31, 47-92 (1998) Zbl 0911.14008 MR 1604294

[HaZu87] Hain, R., Zucker, S.: Unipotent variations of mixed Hodge structures. Invent. Math. 88, 83-124 (1987) Zbl 0622.14007 MR 0877008

[LuMa85] Lubotzky, A., Magid, A.: Varieties of representations of finitely generated groups. Mem. Amer. Math. Soc. 58, no. 336 (1985) Zbl 0598.14042 MR 0818915

[Man99] Manetti, M.: Deformation theory via differential graded algebras. In: Seminari di Geometria Algebrica 1998-1999, Scuola Norm. Sup., 21-48 (1999) MR 1754793

[Pen02] Penacchio, O.: Structures de Hodge mixtes et faisceaux réflexifs semistables. C. R. Math. Acad. Sci. Paris 335, 475-480 (2002) Zbl 1030.14009 MR 1937116

[Pri04] Pridham, J.: The deformation theory of representations of the fundamental group of a smooth variety. arxiv:math/0401344 (2004)

[Pri06] Pridham, J.: Non-abelian real Hodge theory for proper varieties. arxiv/math:0611683 (2006)

[Pri09] Pridham, J.: Formality and splitting of real non-abelian mixed Hodge structures. arxiv/math: 0902.0770 (2009)

[Sch69] Schlessinger, M.: Functors of Artin rings. Trans. Amer. Math. Soc. 130, 208-222 (1968) Zbl 0167.49503 MR 0217093

[Rim72] Rim, D. S.: Formal deformation theory. In: Groupes de Monodromie en Géométrie Algébrique (SGA 7 I), exp. VI, Lecture Notes in Math. 288, Springer, 32-132 (1972) Zbl 0246.14001

[Rim80] Rim, D. S.: Equivariant G-structure on versal deformations. Trans. Amer. Math. Soc. 257, 217-226 (1980) Zbl 0456.14004 MR 0549162

[Sim88] Simpson, C.: Constructing variations of Hodge structure using Yang-Mills theory and applications to uniformization. J. Amer. Math. Soc. 1, 867-918(1988) Zbl 0669.58008 MR 0944577

[Sim92] Simpson, C.: Higgs bundles and local systems. Publ. Math. IHES 75, 5-95 (1992) Zbl 0814.32003 MR 1179076

[Sim94] Simpson, C.: Moduli of representations of the fundamental group of a smooth projective variety. I, Publ. Math. IHES 79, 47-129 (1994); II, Publ. Math. IHES 80, 5-79 (1994) Zbl 0891.14005 Zbl 0891.14006 MR 1307297 MR 1320603

[Sim93] Simpson, C.: Subspaces of moduli spaces of rank one local systems. Ann. Sci. École Norm. Sup. 26, 361-401 (1993) Zbl 0798.14005 MR 1222278

[Sim97] Simpson, C.: Mixed twistor structures. arXiv:alg-geom/9705006 (1997)

[Usu83] Usui, S.: Variation of mixed Hodge structures arising from family of logarithmic deformations. Ann. Sci. École Norm. Sup. 16, 91-107 (1983); II, Classifying space. Duke Math. J. 51, 851-857 (1984) Zbl 0516.14006 Zbl 0558.14005 MR 0719764 MR 0771384

[Zu79] Zucker, S.: Hodge theory with degenerating coefficients. Ann. of Math. 109, 415-476 (1979) Zbl 0446.14002 MR 0534758 